\title{
Orthogonality and Completeness of the Bethe Ansatz Eigenstates of the Nonlinear Schroedinger Model
}

\author{
T.C. Dorlas \\ University College Swansea, Singleton Park, Swansea SA2 8PP, U.K.
}

Received July 20, 1992; in revised form October 26, 1992

\begin{abstract}
A rigorous proof is given of the orthogonality and the completeness of the Bethe Ansatz eigenstates of the $N$-body Hamiltonian of the nonlinear Schroedinger model on a finite interval. The completeness proof is based on ideas of C.N. Yang and C.P. Yang, but their continuity argument at infinite coupling is replaced by operator monotonicity at zero coupling. The orthogonality proof uses the algebraic Bethe Ansatz method or inverse scattering method applied to a lattice approximation introduced by Izergin and Korepin. The latter model is defined in terms of monodromy matrices without writing down an explicit Hamiltonian. It is shown that the eigenfunctions of the transfer matrices for this model converge to the Bethe Ansatz eigenstates of the nonlinear Schroedinger model.
\end{abstract}

\section{Introduction}

The nonlinear Schroedinger model was introduced by Lieb and Liniger [26] in 1963 as the first model of a boson gas that contains a nontrivial adjustable parameter and which can be fully analysed without making approximations. Earlier, Girardeau [20] had introduced a simpler model of a gas of impenetrable bosons in one dimension, but this model behaves effectively as a very high-density gas. Also, the latter model does not have a nontrivial parameter. In many other respects, however, the two models are quite similar and, indeed, Girardeau's model can be obtained from the nonlinear Schroedinger model in the infinite-coupling limit.

In [26], Lieb and Liniger obtained the eigenfunctions of the nonlinear Schroedinger model Hamiltonian with periodic boundary conditions using an "Ansatz" similar to the one used by Bethe in his analysis [11] of the onedimensional Heisenberg model. They did not prove. however, that the set of eigenfunctions thus obtained is actually complete. Many other models have since been shown to be soluble by means of the Bethe Ansatz method and generalisations thereof. (See for example $[19,6,18,34$ and 14].) In particular Baxter's solution of the anisotropic Heisenberg chain [5] was a major breakthrough. Another 
important development was the quantum inverse spectral transform method by the Leningrad group of Faddeev [31] and [16-18] (see also Thacker \& Wilkinson [33]). Concerning the completeness problem, considerable progress was made by Babbitt and Thomas [35] and [1-3]. They prove orthogonality and completeness of the Bethe Ansatz eigenstates for the ground state representation of the Heisenberg chain in the infinite volume limit. In the Heisenberg chain the existence of bound states presents a problem in finite volume. Indeed, in Bethe's original paper the bound states are described only in the infinite volume limit, in which case they are given by the so-called string solutions to the Bethe Ansatz equations. In finite volume this string hypothesis has been shown to be incorrect [13].

Given the eigenvalues of the $N$-body Hamiltonian of a soluble quantum model it is possible to obtain the thermodynamics of the model by means of a method introduced by Yang \& Yang in the case of the nonlinear Schroedinger model in their remarkable paper [36]. This method is analogous to the usual method (see e.g. [25]) for deriving the thermodynamics of the free boson gas. As the authors point out themselves, however, their analysis is not mathematically rigorous. Recently, Yang \& Yang's derivation was put on a rigorous footing in the paper [15] using techniques from large deviation theory. These techniques were developed earlier in [7] for various models of an interacting boson gas involving only the free-gas particle number operators. (See also [8 and 9].) The analysis starts from one basic assumption which was still unproven: the completeness of the Bethe Ansatz eigenstates. In this paper we shall prove this long-standing conjecture, thus completing the analysis of [15].

Let us now briefly outline the way this result is proved in this paper. The $N$-particle Hamiltonian of the nonlinear Schroedinger model is given heuristically by

$$
H_{\kappa}^{N}=-\sum_{j=1}^{N} \frac{\partial^{2}}{\partial x_{j}^{2}}+2 \kappa \sum_{1 \leqq i<j \leqq N} \delta\left(x_{i}-x_{j}\right),
$$

where $\kappa \geqq 0$. A precise mathematical definition of this Hamiltonian will be given in Sect. 2. It uses the KLMN theorem [28] and a Sobolev inequality. (This is very similar to Example X.3 of [28].) As $\kappa$ decreases to 0 one expects $H_{\kappa}^{N}$ to converge to the free-boson Hamiltonian in some sense, while as $\kappa \rightarrow \infty, H_{\kappa}^{N}$ should tend to the Hamiltonian of Girardeau's model of impenetrable bosons (in the case of point particles). In Sect. 3 we shall prove that this is indeed the case if one interprets the convergence in the strong-resolvent sense [27].

In the same paper mentioned above [36], Yang \& Yang argue that the completeness of the Bethe Ansatz eigenfunctions follows from the fact that they converge to a complete set of eigenfunctions for the impenetrable boson model as $\kappa \rightarrow \infty$. That this argument is faulty can be appreciated considering the following simple counter example: Let $T_{\kappa}$ for $0 \leqq \kappa<\infty$ be the operator of $\ell^{2}(\mathbb{N})$ defined by

$$
\left\{\begin{array}{rlrl}
\left(T_{\kappa} u\right)_{n}= & n u_{n} & & \\
\left(T_{\kappa} u\right)_{k+1}= & {\left[(k+1) \sin ^{2} \alpha+\kappa \cos ^{2} \alpha\right] u_{k+1}} & & \\
& +(k+1-\kappa) \sin \alpha \cos \alpha u_{k+2}, & \\
\left(T_{\kappa} u\right)_{k+2}= & (k+1-\kappa) \sin \alpha \cos \alpha u_{k+1} & & \\
& +\left[\left[(k+1) \cos ^{2} \alpha+\kappa \sin ^{2} \alpha\right] u_{k+2},\right. & & \\
\left(T_{\kappa} u\right)_{n}= & (n-1) u_{n} & & \text { if } n \geqq k+3,
\end{array}\right.
$$


where $k=[\kappa]$, that is $k \leqq \kappa<k+1$, and $\alpha=\frac{\pi}{2}(\kappa-k) ; u=\left(u_{1}, u_{2}, \ldots\right) \in \ell^{2}(\mathbb{N})$.

We can diagonalise $T_{\kappa}$ as follows. Let $e_{1}, e_{2}, \ldots$ be the canonical basis for $\ell^{2}(\mathbb{N})$ and put

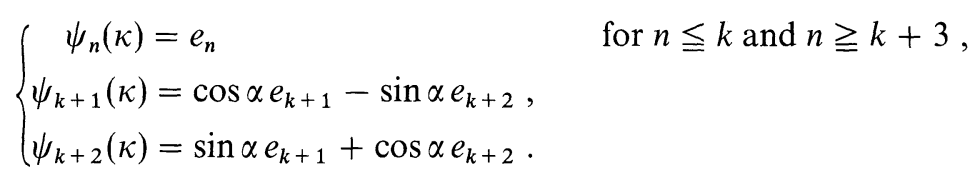

Then

$$
\left\{\begin{aligned}
T_{\kappa} \psi_{n}(\kappa) & =n \psi_{n}(\kappa) & & \text { for } n \leqq k, \\
T_{\kappa} \psi_{k+1}(\kappa) & =\kappa \psi_{k+1}(\kappa), & & \\
T_{\kappa} \psi_{n}(\kappa) & =(n-1) \psi_{n}(\kappa) & & \text { for } n \geqq k+2 .
\end{aligned}\right.
$$

By computing the resolvent $R_{\kappa}(\lambda)=\left(\lambda 1-T_{\kappa}\right)^{-1}$ explicitly it is easy to show that $T_{\kappa}$ is continuous in the strong resolvent sense, and also that $T_{\kappa} \rightarrow T_{\infty}$ as $\kappa \rightarrow \infty$, where

$$
T_{\infty} e_{n}=n e_{n} \quad(n \geqq 1) .
$$

Now consider, for each $\kappa<\infty$, the subset of eigenstates $\left\{\psi_{n}(\kappa)\right\}_{n \neq k+1}$ (the analogue of the Bethe Ansatz eigenstates). This is a continuous set of states. Indeed, as $\kappa \nearrow k+1, \quad \psi_{n}(\kappa)=\psi_{n}(k+1)=e_{n}$ for $n \leqq k$ and $n \geqq k+3$, while $\psi_{k+2}(\kappa) \rightarrow e_{k+1}=\psi_{k+1}(k+1)$. Furthermore, every $e_{n}$ is the limit of $\psi_{n}(\kappa)$ as $\kappa \rightarrow \infty$ and yet $\left\{\psi_{n}(\kappa)\right\}_{n \neq k+1}$ is not complete. (Notice that $\psi_{[\kappa]+1}(\kappa)$ is not continuous, but this does not affect the continuity of $T_{\kappa}$.)

It follows from this example that we have to use some additional information. We shall use the fact that $H_{\kappa}$ is increasing in $\kappa$. Moreover, we consider the limit $\kappa \rightarrow 0$ instead of the limit $\kappa \rightarrow \infty$. This turns out to be easier because $H_{\kappa}^{N}$ is bounded below. In fact it is still an open problem whether it is possible to construct a one-parameter family of operators $T_{\kappa}$ which is increasing and continuous in the strong resolvent sense for $0 \leqq \kappa \leqq \infty$ and such that there exists a continuous family of eigenvectors $\psi_{n}(\kappa)$ such that $\left\{\psi_{n}(\infty)\right\}_{n=1}^{\infty}$ is complete but $\left\{\psi_{n}(\kappa)\right\}_{n=1}^{\infty}$ is not complete for $\kappa<\infty$. In the limit $\kappa \rightarrow 0$ on the other hand we can use the fact that the eigenvalues decrease when $\kappa$ decreases but the number of eigenvalues below an arbitrary number $\lambda$ remains finite. No eigenvalue can therefore escape to infinity and any additional eigenvector other than the Bethe Ansatz eigenvectors would give rise to an extra eigenvalue for $H_{0}^{N}$ below a certain finite $\lambda$. This argument of course uses the independence of the Bethe Ansatz eigenstates. The details are given in Sect. 3.

For the proof of the orthogonality of the Bethe Ansatz eigenstates we make use of the algebraic Bethe Ansatz method developed by Faddeev et al. $[31,17,16]$. This is an alternative method for diagonalising the $N$-body Hamiltonian. (For a comprehensive review of this method applied to various models, see also [14].) To be exact it only works for lattice models. To obtain the eigenstates of a given continuum model one then has to take the continuum limit of an appropriate lattice model approximation. We shall use the lattice model introduced by Izergin and Korepin in [21 and 22]. In Sect. 4 we describe this model in terms of the so-called monodromy matrices, without writing down an explicit Hamiltonian. One can express the Hamiltonian in terms of logarithmic derivatives of the transfer 
matrix $\tau(\lambda)$ and it was shown in [23], using a local gauge transformation, that this Hamiltonian converges to the nonlinear Schroedinger Hamiltonian as the lattice constant tends to zero. It would be interesting to make this derivation rigorous, but we shall not attempt this here. Instead, we show directly that the corresponding eigenstates converge to the Bethe Ansatz eigenstates of the nonlinear Schroedinger model. This proof is presented in Sect. 5. It is rather straightforward but tedious and it involves some delicate cancellations. An alternative approach might be to use the quantum Gelfand-Levitan equation as proposed by Craemer, Thacker and Wilkinson [12]. It should be noted that the proof of convergence presented in this paper also completes Korepin's proof [24] of Gaudin's formula for the norm of the Bethe Ansatz eigenfunctions in the case of the nonlinear Schroedinger model. The advantage of the algebraic Bethe Ansatz method is that it provides us with a one-parameter family of operators which are simultaneously diagonalised. By varying the parameter the eigenvalues corresponding to different eigenstates can be made non-degenerate so that these eigenstates must be orthogonal. This orthogonality proof is presented in Sect. 4, Lemma 4.2.

The proofs for orthogonality and completeness presented in this paper depend strongly on the fact that there are no bound states in this model. For models with bound states the situation is more complicated. Indeed, the solutions of the Bethe Ansatz equations (the analogues of (3.2) and (3.3)) are then manageable only in the thermodynamic limit, where they are arranged in so-called strings in the complex $k$-plane. Under this string hypothesis Takahashi [32] generalized the Yang-Yang thermodynamic formalism to the Heisenberg XXZ chain. Completeness of the Bethe Ansatz eigenstates for the infinite Heisenberg chain in the ground state representation was proved by Babbitt and Thomas [1]. A mathematical proof of the Yang-Yang thermodynamics for this model is, however, still an open problem. The approach of [15] using large deviation theory does not seem hopeful in that case because it requires completeness for the finite chain. Other models may be more amenable to this approach. In particular, the massive Thirring model (see e.g. Bergknoff \& Thacker [10] and Thacker [34]) may be a feasible proposition because it has only a finite number of bound states for a certain range of parameter values.

\section{Definition of the Hamiltonians}

In the following the number of particles $N$ is fixed and will therefore not be included in the notation. We shall use the KLMN Theorem (see [28]) to define the operators $H_{\kappa}$ with $0<\kappa<\infty$ on the Hilbert space $L_{\text {sym }}^{2}\left([0,1]^{N}\right)$ of quadratically integrable symmetric functions on $[0,1]^{N}$ as perturbations of the free gas Hamiltonian $H_{0}$. The latter is defined with periodic boundary conditions as follows: Let

$$
\begin{aligned}
D_{0}=\left\{\psi \in \mathscr{C}_{\text {sym }}^{2}\left([0,1]^{N}\right) \mid \forall x \in[0,1]^{N-1}: \psi(x, 0)\right. & =\psi(x, 1) \text { and } \\
\frac{\partial \psi}{\partial x_{N}}(x, 0) & \left.=\frac{\partial \psi}{\partial x_{N}}(x, 1)\right\},
\end{aligned}
$$


where $C_{\text {sym }}^{2}\left([0,1]^{N}\right)$ denotes the space of twice continuously differentiable symmetric functions. On $D_{0}$ we define the operator $H_{0}$ by

$$
H_{0} \psi=-\Delta \psi \text { for } \psi \in D_{0} .
$$

Then $D_{0}$ contains a complete set of eigenfunctions for $H_{0}$. These are given by

$$
\psi_{\left\{n_{j}\right\}}^{0}(x)=\sum_{\sigma \in \mathscr{S}_{N}} \prod_{i=1}^{N} \exp \left[2 \pi i n_{i} x_{\sigma(i)}\right]
$$

where $x \in[0,1]^{N}$ and $n_{j} \in \mathbb{Z}$ for $j=1,2, \ldots, N$.

Proposition 2.1. The set $\left\{\psi_{\left\{n_{3}\right\}}^{0}\right\}_{n_{1} \leqq n_{2} \leqq \ldots \leqq n_{N}}$ is a total set in the Hilbert space $L_{\text {sym }}^{2}\left([0,1]^{N}\right)$ satisfying

$$
H_{0} \psi_{\left\{n_{j}\right\}}^{0}=(2 \pi)^{2} \sum_{j=1}^{N} n_{j}^{2} \psi_{\left\{n_{j}\right\}}^{0} .
$$

The operator $H_{0}$ defined by (2.2) on $D_{0}$ is therefore essentially self-adjoint.

Proof. The totality follows from the Stone-Weierstrass Theorem. Indeed, consider the algebra of continuous periodic functions $\psi \in \mathscr{C}_{\text {per }}\left([0,1]^{N}\right)$ of the form

$$
\psi(x)=\sum_{m=1}^{M} A_{m} \exp \left[i\left(k_{1}^{(m)} x_{1}+\cdots+k_{N}^{(m)} x_{N}\right)\right],
$$

where $k_{j}^{(m)}=2 \pi n_{j}^{(m)}$ and $n_{j}^{(m)} \in \mathbb{Z}$. This set is clearly self-adjoint and it contains the constant functions. It also separates points of the torus as follows immediately from the one-dimensional case. This set is therefore dense in $\mathscr{C}_{\text {per }}\left([0,1]^{N}\right)$ for the uniform topology and hence also in $L^{2}\left([0,1]^{N}\right)$ for the $L^{2}$-topology. Now let $\operatorname{Sym}_{N}$ be the symmetrisation operator:

$$
\operatorname{Sym}_{N} \psi\left(x_{1}, \ldots, x_{N}\right)=\frac{1}{\sqrt{N} !} \sum_{\sigma \in \mathscr{S}_{N}} \psi\left(x_{\sigma(1)}, \ldots, x_{\sigma(N)}\right),
$$

where the sum runs over the set $\mathscr{S}_{N}$ of all permutations of $\{1,2, \ldots, N\}$. Then, if $D \subset L^{2}\left([0,1]^{N}\right)$ is dense, $\operatorname{Sym}_{N}(D)$ is dense in $L_{\text {sym }}^{2}\left([0,1]^{N}\right)$. But, if $D$ is the above algebra then $\operatorname{Sym}_{N}(D)$ is the set of functions

$$
\operatorname{Sym}_{N}(D)=\left\{\sum_{m=1}^{M} A_{m} \psi_{\left\{n_{j}^{(m)}\right\}}^{0} \mid M=1,2, \ldots, n_{j}^{(m)} \in \mathbb{Z}, A_{m} \in \mathbb{C}\right\} \subset D_{0} .
$$

The relation (2.4) is trivial and the essential self-adjointness of $H_{0}$ follows from Nelson's analytic vector theorem (see [28], Theorem X.39).

We denote the self-adjoint extension of $H_{0}$ also by $H_{0}$. Next we define quadratic forms $h_{\kappa}$ corresponding to the operators $H_{\kappa}$ using the KLMN Theorem ([28], Theorem X.17). For $\phi, \psi \in D_{0}$ we define $h_{\kappa}(\phi, \psi)$ by

$$
h_{\kappa}(\phi, \psi)=\left(\phi \mid H_{0} \psi\right)+\kappa \delta(\phi, \psi),
$$

where

$$
\delta(\phi, \psi)=\left.2 \sum_{1 \leqq i<j \leqq N} \int_{0}^{1} d x_{1} \ldots \widehat{j} \ldots \int_{0}^{1} d x_{N}[\bar{\phi} \psi]\right|_{x_{j}=x_{i}} .
$$


(Here, the symbol $\overparen{j}$ means that the integral over $x_{j}$ is omitted.) To apply the KLMN theorem we must establish the inequality

$$
\delta(\phi, \phi) \leqq c\left\{\left(\phi \mid H_{0} \phi\right)+(\phi \mid \phi)\right\}
$$

for some constant $c>0$ and all $\phi \in D\left(H_{\kappa}\right)$. For $\phi \in D_{0}$ this is a Sobolev inequality which can be derived in the following way: Let $x_{0} \in[0,1]$ be arbitrary, and write

$$
\left.\phi(x)\right|_{x_{j}=x_{1}}=\left.\phi(x)\right|_{x_{j}=x_{0}}+\int_{x_{0}}^{x_{l}} d x_{j} \frac{\partial \phi}{\partial x_{j}} .
$$

Integrating with respect to $x_{0}$ and using the Cauchy-Schwarz inequality we obtain

$$
\begin{aligned}
|\phi(x)|_{x_{j}=x_{\imath}} \mid & \leqq \int_{0}^{1}|\phi(x)| d x_{j}+\int_{0}^{1} d x_{0} \int_{x_{0}}^{x_{l}}\left|\frac{\partial \phi}{\partial x_{j}}\right| d x_{j} \\
& \leqq \int_{0}^{1}|\phi(x)| d x_{j}+\int_{0}^{1}\left|\frac{\partial \phi}{\partial x_{j}}\right| d x_{j} \\
& \leqq\left(\int_{0}^{1}|\phi(x)|^{2} d x_{j}\right)^{1 / 2}+\left(\int_{0}^{1}\left|\frac{\partial \phi}{\partial x_{j}}\right|^{2} d x_{j}\right)^{1 / 2} .
\end{aligned}
$$

Integrating now with respect to the other variables we find

$$
\left.\int_{0}^{1} d x_{1} \ldots \widehat{j} \ldots \int_{0}^{1} d x_{N}|\phi(x)|_{x_{j}=x_{l}}\right|^{2} \leqq 2\left(\int_{[0,1]^{N}} d x|\phi(x)|^{2}+\int_{[0,1]^{N}} d x\left|\frac{\partial \phi}{\partial x_{j}}\right|^{2}\right) .
$$

It follows that

$$
\delta(\phi, \phi) \leqq 2(N-1) \sum_{j=1}^{N} \int_{[0,1]^{N}}\left|\frac{\partial \phi}{\partial x_{j}}\right|^{2} d x+2 N(N-1)(\phi \mid \phi) .
$$

A simple integration by parts shows that the first term on the right-hand side equals $2(N-1)\left(\phi \mid H_{0} \phi\right)$ so that this establishes the inequality (2.8) with $c=2 N(N-1)$ for $\phi \in D_{0}$. But $D_{0}$ is a core for $H_{0}$ and hence also a form core for $h_{0}$. This implies that (2.8) also holds for $\phi \in D\left(H_{0}\right)$. We can now prove:

Proposition 2.2. For all $\kappa \in[0, \infty)$ there exists a unique self-adjoint operator $H_{\kappa}$ with quadratic form domain $Q_{\kappa}=Q_{0}=Q\left(H_{0}\right)$ such that $\left(\phi \mid H_{\kappa} \psi\right)=h_{\kappa}(\phi, \psi)$ for all $\phi, \psi \in Q_{0}$. The operators $H_{\kappa}$ are increasing in $\kappa$.

Proof. We proceed by induction on $\kappa$. Assume that $H_{\kappa_{0}}$ is defined as in the proposition for some $\kappa_{0} \geqq 0$. Then, by (2.12) and the fact that $H_{\kappa_{0}} \geqq H_{0}$,

$$
\delta(\phi, \phi) \leqq 2(N-1) h_{\kappa_{0}}(\phi, \phi)+2 N(N-1)\|\phi\|^{2} .
$$

It follows by the KLMN theorem that $H_{\kappa}$ is uniquely defined for $\kappa<\kappa_{0}+\frac{1}{2(N-1)}$. Obviously, $H_{\kappa} \geqq H_{\kappa_{0}}$ if $\kappa>\kappa_{0}$ as $\delta(\phi, \phi) \geqq 0$.

About the domain of the operators $H_{\kappa}$ we can say the following:

Proposition 2.3. Let $\mathscr{R}$ be the region

$$
\mathscr{R}=\left\{x \in[0,1]^{N} \mid 0 \leqq x_{1} \leqq \ldots \leqq x_{N} \leqq 1\right\}
$$


and define, for $\kappa \in(0, \infty)$,

$$
\begin{aligned}
& D_{\kappa}=\left\{\phi \in \mathscr{C}_{\mathrm{sym}}\left([0,1]^{N}\right)|\phi|_{\mathscr{R}} \in \mathscr{C}^{2}(\mathscr{R}) \text { and for } u \in \mathscr{R}\right. \\
& \phi\left(0, u_{1}, \ldots, u_{N-1}\right)=\phi\left(u_{1}, \ldots, u_{N-1}, 1\right) \\
& \frac{\partial \phi}{\partial x_{1}}\left(0, u_{1}, \ldots, u_{N-1}\right)=\frac{\partial \phi}{\partial x_{N}}\left(u_{1}, \ldots, u_{N-1}, 1\right) \text { and } \\
&\left.\lim _{u_{i+1} \downarrow u_{i}}\left(\frac{\partial \phi(u)}{\partial u_{i+1}}-\frac{\partial \phi(u)}{\partial u_{i}}\right)=\left.\kappa \phi(u)\right|_{u_{i+1}=u_{i}}\right\} .
\end{aligned}
$$

Then $D_{\kappa} \subset D\left(H_{\kappa}\right)$ and

$$
H_{\kappa} \phi=-\Delta \phi \quad \text { for } \phi \in D_{\kappa} .
$$

Proof. Using the fact that

$$
\begin{aligned}
Q_{0}=\left\{\phi \in L_{\text {sym }}^{2}\left([0,1]^{N}\right) \mid\right. & \exists \text { sequence } \phi_{n} \in D_{0}: \phi_{n} \rightarrow \phi \text { in } L^{2} \\
& \text { and } \left.\tilde{h}_{\kappa}\left(\phi_{n}-\phi_{m}\right) \rightarrow 0(n, m \rightarrow \infty)\right\}
\end{aligned}
$$

a simple regularisation shows that $D_{\kappa} \subset Q_{0}$. Now

$$
D\left(H_{\kappa}\right)=\left\{\phi \in Q_{0} \mid \exists \xi \in L_{\text {sym }}^{2}:(\psi \mid \xi)=h_{\kappa}(\psi, \phi) \forall \psi \in Q_{0}\right\} .
$$

A simple integration by parts shows that $h_{\kappa}(\psi, \phi)=-(\psi \mid \Delta \phi)$ for $\psi \in D_{0}$. This proves that $\phi \in D\left(H_{\kappa}\right)$ because $D_{0}$ is a form core for $H_{\kappa}$.

Remark. The operator $H_{\infty}$ is also well-defined. It is the free particle Hamiltonian with Dirichlet boundary conditions on the lines $x_{i}=x_{j}$. Its quadratic form domain is given by

and

$$
Q_{\infty}=\left\{\phi \in Q_{0} \mid \sup _{\kappa<\infty} h_{\kappa}(\phi, \phi)<\infty\right\}
$$

$$
h_{\infty}(\phi, \phi)=\sup _{\kappa<\infty} h_{\kappa}(\phi, \phi) \text {. }
$$

\section{Completeness of the Bethe Ansatz Eigenstates}

As explained in the introduction we shall prove the completeness of the Bethe Ansatz eigenstates of the Hamiltonians defined in the previous section using a modification of the continuity argument proposed by Yang and Yang in their far-sighted paper [36]. The argument is very general and can be formulated for a general one-parameter family of operators. It assumes the orthogonality of the eigenvectors which will be proved in Sect. 5 .

We begin by defining the Bethe Ansatz eigenfunctions for the nonlinear Schroedinger model. For a given set of wave numbers $k_{1}<k_{2}<\ldots<k_{N}$ one defines functions $\psi_{\left\{k_{j}\right\}}^{B A} \in L_{\mathrm{sym}}^{2}\left([0,1]^{N}\right)$ by

$$
\psi_{\left\{k_{j}\right\}}^{B A}\left(x_{1}, \ldots, x_{N}\right)=\sum_{\sigma \in \mathscr{S}_{N}} A_{\sigma} \exp \left[i \sum_{j=1}^{N} k_{\sigma(j)} x_{j}\right]
$$


for $0 \leqq x_{1} \leqq x_{2} \leqq \ldots \leqq x_{N} \leqq 1$, where $\mathscr{S}_{N}$ is the set of permutations of $\{1, \ldots, N\}$. It was shown in [26] that these functions are eigenfunctions of $H_{\kappa}$ provided that the wave numbers $k_{1}, \ldots, k_{N}$ and the coefficients $A_{\sigma}$ satisfy the following conditions:

$$
k_{j}=2 \pi m_{j}-\sum_{i=1}^{N} \theta_{\kappa}\left(k_{j}-k_{i}\right)
$$

if $N$ is odd and

$$
k_{j}=2 \pi\left(m_{j}+\frac{1}{2}\right)-\sum_{i=1}^{N} \theta_{\kappa}\left(k_{j}-k_{i}\right),
$$

if $N$ is even, where $m_{j} \in \mathbb{Z}$ and the function $\theta_{\kappa}$ is given by

$$
\theta_{\kappa}(k)=2 \arctan (k / \kappa)
$$

and

$$
\frac{A_{\tau}}{A_{\sigma}}=-\exp \left[-i \theta_{\kappa}\left(k_{\tau(j)}-k_{\sigma(j)}\right)\right]
$$

whenever $\tau$ and $\sigma$ differ by a single transposition:

$$
\tau(j)=\sigma(j+1) ; \quad \tau(j+1)=\sigma(j) \text { and } \tau(i)=\sigma(i) \text { for } i \neq j, j+1 .
$$

One easily proves following [26]:

Lemma 3.1. If the set of wave numbers $\left\{k_{1}, \ldots, k_{N}\right\}$ satisfies the conditions (3.2) respectively (3.3) with $m_{j} \in \mathbb{Z}$ and the coefficients $A_{\sigma}$ are defined by (3.4) then $\psi_{\left\{k_{j}\right\}}^{B A} \in D\left(H_{\kappa}\right)$ and

$$
H_{\kappa} \psi_{\left\{k_{j}\right\}}^{B A}=\left(\sum_{j=1}^{N} k_{j}^{2}\right) \psi_{\left\{k_{j}\right\}}^{B A} .
$$

In [36] (see also [15], Prop. 5.1) it was proved that there is exactly one Bethe Ansatz eigenstate for every set of integers $m_{1}<\cdots<m_{N}$ :

Lemma 3.2. For every set of integers $m_{1}<\cdots<m_{N}$ there exists a unique solution $k_{1}<\cdots<k_{N}$ to Eqs. (3.2) respectively (3.3). Moreover, these solutions $\left\{k_{j}(\kappa)\right\}$ depend continuously on $\kappa \in(0, \infty)$.

Proof. The first statement was proved by Yang and Yang in [36]. They define a function

$$
B\left(k_{1}, \ldots, k_{N}\right)=\frac{1}{2} \sum_{j=1}^{N} k_{j}^{2}-2 \pi \sum_{j=1}^{N} I_{j} k_{j}+\frac{1}{2} \sum_{i, j=1}^{N} \Theta_{\kappa}\left(k_{i}-k_{j}\right),
$$

where

$$
I_{j}= \begin{cases}m_{j} & \text { if } N \text { is odd } \\ m_{j}+\frac{1}{2} & \text { if } N \text { is even }\end{cases}
$$

and

$$
\Theta_{\kappa}(k)=\int_{0}^{k} \theta_{\kappa}\left(k^{\prime}\right) d k^{\prime}
$$


They then showed that $\left(k_{1}, \ldots, k_{N}\right)$ minimises $B$ if and only if it satisfies (3.2) respectively (3.3), and that $B$ is strictly convex. Notice, however, that $B$ is also continuously differentiable as a function of $\kappa$. The minimiser therefore varies continuously with $\kappa$.

In this section we shall prove that the functions $\psi_{\left\{k_{j}\right\}}^{B A}$ are complete in $L_{\text {sym }}^{2}\left([0,1]^{N}\right)$ assuming that they are orthogonal. First we note the following:

Lemma 3.3. Let $\left\{\psi_{\left\{n_{j}\right\}}^{0}\right\}_{n_{1} \leqq \ldots \leqq n_{N}}$ be the total set of eigenfunctions of $H_{0}$ defined in (2.3). Then, for every set of integers $n_{1} \leqq \cdots \leqq n_{N}$ there exists a set of integers $m_{1}<\cdots<m_{N}$ such that

$$
\psi_{\left\{k_{j}\right\}}^{B A} \rightarrow \psi_{\left\{n_{j}\right\}}^{0} \text { as } \kappa \rightarrow 0
$$

where $k_{1}<\cdots<k_{N}$ are the solutions of (3.2) if $N$ is odd and (3.3) if $N$ is even.

Proof. Let $n_{1} \leqq \cdots \leqq n_{N}$ be given. If $N$ is odd then we define $m_{j}$ as follows:

$$
m_{j}=n_{j}-\frac{N+1}{2}+j
$$

if $N$ is even then we define

$$
m_{j}=n_{j}-\frac{N}{2}+j-1
$$

Both cases are similar; we consider the case that $N$ is odd. Then

$$
k_{j} \rightarrow 2 \pi m_{j}-\sum_{i=1}^{j-1}(+\pi)-\sum_{i=j+1}^{N}(-\pi)=2 \pi m_{j}+(N-2 j+1) \pi=2 \pi n_{j},
$$

and

$$
\frac{A_{\tau}}{A_{\sigma}} \rightarrow-\exp \left[-i \pi \varepsilon\left(k_{\tau(j)}-k_{\sigma(j)}\right]=1\right.
$$

Lemma 3.4. For all $c_{0} \in[0, \infty)$,

$$
\tilde{h}_{\kappa_{0}}(\phi)=\inf _{\kappa>\kappa_{0}} \tilde{h}_{\kappa}(\phi) \quad \forall \phi \in Q_{0} .
$$

Proof. It is obvious that the equality holds if $\phi \in D_{0}$. But $D_{0}$ is a form core for $H_{0}$. This means that $D_{0}$ is dense in $Q_{0}$ for the norm $\|\phi\|_{+}^{2}=\|\phi\|^{2}+\left(\phi \mid H_{0} \phi\right)$. By the inequality (2.8), if $\phi_{n} \rightarrow \phi$ with respect to this + -norm then $\tilde{h}_{\kappa}\left(\phi_{n}\right) \rightarrow \tilde{h}_{\kappa}(\phi)$ uniformly in $\kappa$ on bounded intervals.

Corollary. For all $\kappa_{0} \in[0, \infty), H_{\kappa_{0}}=\lim _{\kappa_{\downarrow} \kappa_{0}} H_{\kappa}$ in the strong resolvent sense.

Proof. This follows immediately from [27], Theorem S 16 or [30].

Lemma 3.5. Let $\mu_{n}(\kappa)$ be the $n^{\text {th }}$ eigenvalue of $H_{\kappa}$. Then

$$
\mu_{n}\left(\kappa_{0}\right)=\inf _{\kappa>\kappa_{0}} \mu_{n}(\kappa) \quad\left(\kappa_{0} \in[0, \infty)\right)
$$


Proof. By the Min-Max Theorem (Theorem XIII.2 of [29]),

$$
\mu_{n}(\kappa)=\sup _{\substack{\psi \in Q_{0}:\|\psi\|=1 \\ \phi_{1}, \ldots, \phi_{n-1} \\ \psi \perp\left[\phi_{1}, \ldots, \phi_{n-1}\right]}} \tilde{h}_{\kappa}(\psi) .
$$

It follows immediately that $\mu_{n}\left(\kappa_{0}\right) \leqq \mu_{n}(\kappa)$ if $\kappa>\kappa_{0}$. Conversely let $\lambda=\mu_{n}\left(\kappa_{0}\right)$ and let $\varepsilon>0$ be arbitrary. We shall show that, if $\kappa-\kappa_{0}$ is small enough then, for all $\phi_{1}, \ldots, \phi_{n-1}$ there exists $\psi \in Q_{0}$ such that $\|\psi\|=1, \psi \perp\left[\phi_{1}, \ldots, \phi_{n-1}\right]$ and $\tilde{h_{\kappa}}(\psi)<\lambda+\varepsilon$. Indeed, by (3.12) there exists $\psi \in Q_{0}$ with $\|\psi\|=1$ and $\psi \perp\left[\phi_{1}, \ldots, \phi_{n-1}\right]$ such that $\tilde{h}_{\kappa_{0}}(\psi)<\lambda+\varepsilon / 2$. By Eq. $(2.8), \tilde{h}_{\kappa}(\psi) \leqq \tilde{h}_{\kappa_{0}}(\psi)$ $+c\left(\kappa-\kappa_{0}\right)\left(1+\tilde{h}_{\kappa_{0}}(\psi)\right)$, so if we choose $c\left(\kappa-\kappa_{0}\right)(2+\lambda)<\varepsilon / 2$ then (assuming $\varepsilon<2$ ) we have $\tilde{h}_{\kappa}(\psi)<\lambda+\varepsilon$ as required.

Corollary. The operators $H_{\kappa}$ have purely discrete spectrum for all $\kappa \in[0, \infty)$ and their resolvents are compact operators.

Proof. This follows immediately from Lemma 3.5 and Theorem XIII.64 of [29].

Remark. This result can be used to strengthen the corollary of Lemma 3.4 as follows. Define the operators $R_{\kappa}=\left(1+H_{\kappa}\right)^{-1}$. Then $R_{\kappa} \rightarrow R_{\kappa_{0}}$ strongly. Furthermore, $R_{\kappa}$ is compact for all $\kappa$. It follows that, in particular $\left(R_{\kappa_{0}}\right)^{1 / 2}$ and $\left(R_{\kappa_{0}}\right)^{1 / 4}$ are compact. (See [27], Problem VI.46.) Now choose $\varepsilon>0$ arbitrarily small. Then, since the unit ball $B_{1}$ of $L_{\text {sym }}^{2}$ is mapped onto a compact set by $\left(R_{\kappa_{0}}\right)^{1 / 4}$, there exists a finite subset $\psi_{1}, \ldots, \psi_{M}$ of $B_{1}$ such that for all $\psi \in B_{1}$ there exists $\psi_{m}$ with $\left\|\left(R_{\kappa_{0}}\right)^{1 / 4}\left(\psi-\psi_{m}\right)\right\|<\varepsilon$. Now,

$$
\left\|R_{\kappa}\left(R_{\kappa_{0}}\right)^{-1 / 4}\right\| \leqq\left\|\left(R_{\kappa_{0}}\right)^{1 / 4}\right\|\left\|\left(R_{\kappa_{0}}\right)^{-1 / 4} R_{\kappa}\left(R_{\kappa_{0}}\right)^{-1 / 4}\right\| \leqq 1,
$$

so if we let $\kappa-\kappa_{0}$ be small enough so that $\left\|\left(R_{\kappa}-R_{\kappa_{0}}\right) \psi_{m}\right\|<\varepsilon$ for all $m=1, \ldots, M$ then

$$
\begin{aligned}
\left\|\left(R_{\kappa}-R_{\kappa_{0}}\right) \psi\right\| \leqq & \left\|R_{\kappa}\left(R_{\kappa_{0}}\right)^{-1 / 4}\left(\left(R_{\kappa_{0}}\right)^{1 / 4} \psi-\left(R_{\kappa_{0}}\right)^{1 / 4} \psi_{m}\right)\right\| \\
& +\left\|\left(R_{\kappa}-R_{\kappa_{0}}\right) \psi_{m}\right\|+\left\|R_{\kappa_{0}}\left(\psi-\psi_{m}\right)\right\|<3 \varepsilon .
\end{aligned}
$$

This proves that $\left\|R_{\kappa}-R_{\kappa_{0}}\right\| \rightarrow 0$ as $\kappa \downarrow \kappa_{0}$ and hence that $H_{\kappa} \rightarrow H_{\kappa_{0}}$ in norm resolvent sense!

We now order the Bethe Ansatz eigenstates $\psi_{n}^{B A}(\kappa)$ so that the eigenvalues $\lambda_{n}(\kappa)=\sum_{j=1}^{N} k_{j}(\kappa)^{2}$ satisfy $\lambda_{n}(\kappa) \leqq \lambda_{n+1}(\kappa)$.

Lemma 3.6. Suppose that, for some $m, \mu_{m}(\kappa)<\lambda_{m}(\kappa)$. Then $\mu_{m+k+1}(\kappa) \leqq \lambda_{m+k}(\kappa)$ for all $k \geqq 0$.

Proof. This is obvious since $\mu_{m+k+1}$ is the $(k+1)^{\text {th }}$ eigenvalue above $\mu_{m}$ and there are at least $k+1$ eigenvalues between $\mu_{m}$ and $\lambda_{m+k}$, namely $\lambda_{m}, \ldots, \lambda_{m+k}$.

Theorem 3.1. Assume that the Bethe Ansatz eigenstates $\psi_{\left\{\boldsymbol{k}_{j}\right\}}^{B A}$ defined by (3.1), where the wave numbers $\left\{k_{j}\right\}_{j=1}^{N}$ satisfy (3.2) if $N$ is odd and (3.3) if $N$ is even, and where the coefficients $A_{\sigma}$ satisfy (3.4), are mutually orthogonal. Then they form a complete set in $L_{\text {sym }}^{2}\left([0,1]^{N}\right)$ as $m_{1}<m_{2}<\cdots<m_{N}$ run through $\mathbb{Z}$.

Proof. We shall prove that, for all $\kappa<\infty$ and all $n=1,2, \ldots, \mu_{n}(\kappa)=\lambda_{n}(\kappa)$. Indeed, suppose that for some $\kappa_{0}$ and some positive integer $n_{0}, \mu_{n_{0}}\left(\kappa_{0}\right)<\lambda_{n_{0}}\left(\kappa_{0}\right)$. 
Since $\lambda_{n}(0) \rightarrow \infty$ as $n \rightarrow \infty$, there exists $p$ such that $\mu_{n_{0}}\left(\kappa_{0}\right)<\lambda_{p}(0)$. Now define

$$
\kappa_{1}=\inf \left\{\kappa>0 \mid \mu_{n_{0}}(\kappa)<\lambda_{n_{0}}(\kappa)\right\} .
$$

Then there exists a sequence $\kappa_{1}^{(n)} \searrow \kappa_{1}$ such that $\mu_{n_{0}}\left(\kappa_{1}^{(n)}\right)<\lambda_{n_{0}}\left(\kappa_{1}^{(n)}\right)$ and hence, by Lemma 3.6,

$$
\mu_{n_{0}+k+1}\left(\kappa_{1}^{(n)}\right) \leqq \lambda_{n_{0}+k}\left(\kappa_{1}^{(n)}\right) \quad \forall k \geqq 0 .
$$

Taking $n \rightarrow \infty$ we find, using Lemma 3.5 and the continuity of the Bethe Ansatz eigenvalues $\lambda_{m}(\kappa)$ which follows from Lemma 3.2 and the assumed orthogonality,

$$
\mu_{n_{0}+k+1}\left(\kappa_{1}\right) \leqq \lambda_{n_{0}+k}\left(\kappa_{1}\right) \quad \forall k \geqq 0 .
$$

Let $n_{1}>n_{0}$ be the smallest integer such that $\lambda_{n_{1}}\left(\kappa_{1}\right)>\lambda_{n_{0}}\left(\kappa_{1}\right)$. Then $\mu_{n_{1}}\left(\kappa_{1}\right) \leqq \lambda_{n_{1}-1}\left(\kappa_{1}\right)=\lambda_{n_{0}}\left(\kappa_{1}\right)<\lambda_{n_{1}}\left(\kappa_{1}\right)$ and by continuity, $\mu_{n_{1}}\left(\kappa_{1}\right) \leqq \lambda_{n_{0}}\left(\kappa_{1}\right)=$ $\mu_{n_{0}}\left(\kappa_{1}\right) \leqq \mu_{n_{0}}\left(\kappa_{0}\right)<\lambda_{p}(0)$. Proceeding in this way we can define sequences $\left(\kappa_{k}\right)$ and $\left(n_{k}\right)$ by

and

$$
\kappa_{k}=\inf \left\{\kappa>0 \mid \mu_{n_{k-1}}(\kappa)<\lambda_{n_{k-1}}(\kappa)\right\}
$$

$$
n_{k}=\min \left\{n>n_{k-1} \mid \lambda_{n}\left(\kappa_{k}\right)>\lambda_{n_{k-1}}\left(\kappa_{k}\right)\right\} \text {. }
$$

The sequences stop when $\kappa_{k}=0$. We now argue that there must be a $k$ such that $\kappa_{k}=0$. Indeed, for $\kappa<\kappa_{k}, \quad \mu_{n_{k-1}}(\kappa)=\lambda_{n_{k-1}}(\kappa)$ and in particular, $\mu_{n_{k-1}}(0)=\lambda_{n_{k-1}}(0)$. On the other hand, $\mu_{n_{k-1}}(0) \leqq \mu_{n_{k-1}}\left(\kappa_{k-1}\right)<\lambda_{p}(0)$ so that $n_{k-1}<p$, i.e. the sequence $\left(n_{k}\right)$ is bounded. It must therefore break off and we conclude that $\kappa_{k}=0$ for some $k$ so that $\mu_{n_{k-1}}(\kappa)<\lambda_{n_{k-1}}(\kappa)$ for all $\kappa>0$ and hence $\mu_{n_{k-1}+1}(0) \leqq \lambda_{n_{k-1}}(0)$. This contradicts Proposition 2.1.

In Sect. 5 we shall prove the orthogonality of the Bethe Ansatz eigenstates, thus completing the proof of the completeness of these states.

Remark. Notice that the proof of this theorem uses only Lemma 3.5. It follows from the argument of the remark following Lemma 3.5 and Theorem VIII.23 of [27] that this lemma holds in the general situation of a continuous, non-decreasing one-parameter family $H_{\kappa}$ of positive operators with compact resolvent. The latter condition actually follows if $H_{0}$ has compact resolvent as in the corollary of Lemma 3.5. We can therefore generalise Theorem 3.1 to

Theorem 3.2. Let $\left\{H_{\kappa}\right\}_{\kappa} \geq 0$ be a one-parameter family of positive self-adjoint operators on a Hilbert space $\mathscr{H}$. Assume that $\kappa \mapsto H_{\kappa}$ is monotonically nondecreasing and continuous in the strong resolvent sense and that $H_{0}$ has compact resolvent. Suppose further that there exists a set of linearly independent eigenfunctions $\left\{\phi_{n}(\kappa)\right\}$ for $H_{\kappa}$ which depends continuously on $\kappa$ and is complete at $\kappa=0$. Then this set is complete in $\mathscr{H}$ for all $\kappa \geqq 0$.

\section{Lattice Approximation and the Algebraic Bethe Ansatz}

We shall prove the orthogonality of the Bethe Ansatz eigenstates (3.1) by means of a lattice approximation introduced by Korepin and Izergin [21]. This lattice model is defined by a commuting set of transfer matrices which can be diagonalised by means of the so-called algebraic Bethe Ansatz introduced by Faddeev et al. (see [16]). Although the corresponding Hamiltonian is a highly complicated object we 
shall prove directly that the corresponding eigenstates converge to the Bethe Ansatz eigenstates of the nonlinear Schroedinger model as the lattice constant tends to zero. Using the fact that the eigenstates of the lattice model are simultaneous eigenstates of a one-parameter family of operators one can prove that they are orthogonal. Taking the continuum limit it then follows that the Bethe Ansatz states (3.1) are also orthogonal.

In this section we shall outline the algebraic Bethe Ansatz method, also called the quantum inverse scattering method, for the special case of the Korepin-Izergin model. More extensive reviews can be found in [16, 18 and 14]. It should be noted that the method was originally developed in the case of the nonlinear Schroedinger model itself $[31,17]$. However, in that case the method is only approximate. It is the lattice approximation introduced by Korepin and Izergin [21] which is exactly diagonalisable by this method. They proposed a Hamiltonian for their model in the form of a trace formula analogous to the one discovered by Baxter in the case of the one-dimensional Heisenberg model [5]. In [23] an argument is presented using a local gauge transformation which shows that this Hamiltonian approaches the nonlinear Schroedinger Hamiltonian in the continuum limit. The mathematical status of this argument has yet to be investigated. To define the transfer matrices of the Korepin-Izergin lattice model we subdivide the interval $[0,1]$ into $M$ intervals of length $\Delta=1 / M$. In the following $\Delta$ will be fixed and we impose periodic boundary conditions by identifying the lattice points 0 and 1 . At each lattice point $x_{n}=n \Delta(n=1, \ldots, M)$ we assume given creation and annihilation operators $a_{n}^{*}$ and $a_{n}$ operating on a local Hilbert space $\mathscr{H}_{n} \approx \mathscr{H}$ satisfying the commutation relations

$$
\left[a_{n}, a_{m}^{*}\right]=\Delta \delta_{n m} .
$$

(The unusual factor $\Delta$ is introduced so that we can consider $a_{n}$ and $a_{n}^{*}$ as the continuum annihilation and creation operators $a$ and $a^{*}$ applied to characteristic functions: $a_{n}=a\left(1_{\left[x_{n-1}, x_{n}\right]}\right)$ and similarly $a_{n}^{*}=a^{*}\left(1_{\left[x_{n-1}, x_{n}\right]}\right)$.) Let $\mathscr{F}^{\Delta}=\bigoplus_{N=1}^{\infty} \mathscr{F}_{N}^{A}$ be the corresponding Fock space where $\mathscr{F}_{N}^{\Delta}=\left(\otimes_{k=1}^{N} \mathscr{H}\right)_{\text {sym }}$ is the symmetrised tensor product of $N$ copies of $\mathscr{H}$. A general element $\Psi \in \mathscr{F}_{N}^{\Delta}$ can be written as

$$
\Psi=\sum_{1 \leqq n_{1} \leqq \ldots \leqq n_{N} \leqq M} f\left(n_{1}, \ldots, n_{N}\right) a_{n_{1}}^{*} \ldots a_{n_{N}}^{*} \Omega,
$$

where $\Omega=\otimes_{n=1}^{M} \Omega_{n}$ is the tensor product of the ground states of the local oscillators: $a_{n} \Omega_{n}=0$. Its norm is given by

where

$$
\|\Psi\|^{2}=\Delta^{N} \sum_{1 \leqq n_{1} \leqq \ldots \leqq n_{N} \leqq M}\left|f\left(n_{1}, \ldots, n_{N}\right)\right|^{2} \theta\left(n_{1}, \ldots, n_{N}\right),
$$

$$
\theta\left(n_{1}, \ldots, n_{N}\right)=\sum_{\pi \in \mathscr{P}(N)} \prod_{P \in \pi}(|P|-1) ! \delta_{n P} .
$$

(Here $\mathscr{P}(N)$ denotes the set of partitions of $\{1, \ldots, N\}$ and $\delta_{n P}=\prod_{i \in P ; i \neq j} \delta_{n_{l}, n_{J}}$ if $j \in P \subset\{1, \ldots, N\}$.) These formulae will be useful in Sect. 5. Following Faddeev [16] and Korepin and Izergin [21] we now define local $2 \times 2$-matrices $L_{n}(\lambda)$ for $\lambda \in \mathbb{R}$ with operator-valued entries, the so-called monodromy matrices, as follows:

$$
L_{n}(\lambda)=\left(\begin{array}{cc}
\alpha_{n}(\lambda) & \beta_{n}^{*} \\
\beta_{n} & \alpha_{n}^{*}(\lambda)
\end{array}\right),
$$


where $\alpha_{n}(\lambda)$ and $\beta_{n}^{*}(\lambda)$ are the operators

and

$$
\alpha_{n}(\lambda)=\left(1-\frac{1}{2} i \lambda \Delta\right) \mathbf{1}+\frac{1}{2} \kappa a_{n}^{*} a_{n}
$$

with

$$
\beta_{n}^{*}=-i \sqrt{\kappa a_{n}^{*}} \rho_{n}
$$

$$
\rho_{n}=\left(1+\frac{1}{4} \kappa a_{n}^{*} a_{n}\right)^{1 / 2}
$$

The transfer matrix $\tau(\lambda)$ of the model is now defined as follows: We form the product of matrices

$$
T_{M}(\lambda)=L_{M}(\lambda) \ldots L_{1}(\lambda)=\left(\begin{array}{ll}
A_{M}(\lambda) & B_{M}^{*}(\lambda) \\
B_{M}(\lambda) & A_{M}^{*}(\lambda)
\end{array}\right)
$$

and take the two-dimensional partial trace

$$
\tau_{M}(\lambda)=\operatorname{Tr}_{2}\left(L_{M}(\lambda) \ldots L_{1}(\lambda)\right)=A_{M}(\lambda)+A_{M}^{*}(\lambda) .
$$

The main result of the algebraic Bethe Ansatz method is:

Theorem 4.1. Define for any real number $\lambda$,

$$
\alpha(\lambda)=1-i \lambda \Delta / 2
$$

and suppose that $\lambda_{1}<\lambda_{2}<\ldots<\lambda_{N}$ are real numbers satisfying the coupled nonlinear equations

$$
\left(\frac{\alpha\left(\lambda_{k}\right)}{\bar{\alpha}\left(\lambda_{k}\right)}\right)^{M}=\prod_{l \neq k} \frac{\lambda_{k}-\lambda_{l}-i \kappa}{\lambda_{k}-\lambda_{l}+i \kappa} .
$$

Then the wave function

$$
\Psi\left(\lambda_{1}, \ldots, \lambda_{N}\right)=B_{M}^{*}\left(\lambda_{1}\right) \ldots B_{M}^{*}\left(\lambda_{N}\right) \Omega
$$

is an eigenfunction of $\tau_{M}(\lambda)$ for every $\lambda \in \mathbb{R}$ with eigenvalue given by

$$
E_{M}\left(\lambda ; \lambda_{1}, \ldots, \lambda_{N}\right)=\prod_{k=1}^{N} \frac{\lambda_{k}-\lambda-i \kappa}{\lambda_{k}-\lambda} \alpha(\lambda)^{M}+\prod_{k=1}^{N} \frac{\lambda-\lambda_{k}-i \kappa}{\lambda-\lambda_{k}} \bar{\alpha}(\lambda)^{M} .
$$

Proof. We begin by defining

and

$$
b(\lambda, \mu)=\frac{\lambda-\mu}{\lambda-\mu-i \kappa}
$$

$$
c(\lambda, \mu)=\frac{-i \kappa}{\lambda-\mu-i \kappa} .
$$

The quantum $R$-matrix is the $4 \times 4$ scalar matrix given by

$$
R(\lambda, \mu)=\left(\begin{array}{cccc}
1 & 0 & 0 & 0 \\
0 & b(\lambda, \mu) & c(\lambda, \mu) & 0 \\
0 & c(\lambda, \mu) & b(\lambda, \mu) & 0 \\
0 & 0 & 0 & 1
\end{array}\right)
$$


It is an easy but tedious exercise to show that it satisfies the following commutation relations, sometimes called the star-triangle relations:

$$
R(\lambda, \mu)\left(L_{n}(\lambda) \otimes L_{n}(\mu)\right)=\left(1 \otimes L_{n}(\mu)\right)\left(L_{n}(\lambda) \otimes 1\right) R(\lambda, \mu),
$$

where the tensor product of $2 \times 2$-matrices is defined explicitly by

$$
\mathscr{M} \otimes \mathscr{N}=\left(\begin{array}{cc}
\mathscr{M}_{11} \mathscr{N} & \mathscr{M}_{12} \mathscr{N} \\
\mathscr{M}_{21} \mathscr{N} & \mathscr{M}_{22} \mathscr{N}
\end{array}\right)
$$

Now, by the fact that, for $n \neq m, \alpha_{n}(\lambda)$ and $\beta_{m}$ commute, we have

$$
T_{M}(\lambda) \otimes T_{M}(\mu)=\prod_{n=1}^{M}\left(L_{n}(\lambda) \otimes L_{n}(\mu)\right) \text {. }
$$

By repeated application of (4.18) we therefore obtain

$$
R(\lambda, \mu)\left(T_{M}(\lambda) \otimes T_{M}(\mu)\right)=\left(\mathbf{1} \otimes T_{M}(\mu)\right)\left(T_{M}(\lambda) \otimes \mathbf{1}\right) R(\lambda, \mu) .
$$

These relations imply, in particular,

$$
B_{M}^{*}(\lambda) A_{M}(\mu)=c(\lambda, \mu) B_{M}^{*}(\mu) A_{M}(\lambda)+b(\lambda, \mu) A_{M}(\mu) B_{M}^{*}(\lambda)
$$

which can be written as

$$
A_{M}(\mu) B_{M}^{*}(\lambda)=\frac{1}{b(\lambda, \mu)} B_{M}^{*}(\lambda) A_{M}(\mu)-\frac{c(\lambda, \mu)}{b(\lambda, \mu)} B_{M}^{*}(\mu) A_{M}(\lambda)
$$

provided that $\lambda \neq \mu$, and similarly,

$$
A_{M}^{*}(\lambda) B_{M}^{*}(\mu)=\frac{1}{b(\lambda, \mu)} B_{M}^{*}(\mu) A_{M}^{*}(\lambda)-\frac{c(\lambda, \mu)}{b(\lambda, \mu)} B_{M}^{*}(\lambda) A_{M}^{*}(\mu) .
$$

Another important consequence of the commutations relations (4.20) is that the operators $B_{M}^{*}(\lambda)$ commute:

$$
B_{M}^{*}(\lambda) B_{M}^{*}(\mu)=B_{M}^{*}(\mu) B_{M}^{*}(\lambda) .
$$

These relations enable us to evaluate the operators $A_{M}(\lambda)$ and $A_{M}^{*}(\lambda)$ applied to the vector $\Psi\left(\lambda_{1}, \ldots, \lambda_{N}\right)$ defined by (4.13). In the case $N=1$ we have simply

$$
A_{M}(\lambda) \Psi\left(\lambda_{1}\right)=\frac{1}{b\left(\lambda_{1}, \lambda\right)} B_{M}^{*}\left(\lambda_{1}\right) A_{M}(\lambda) \Omega-\frac{c\left(\lambda_{1}, \lambda\right)}{b\left(\lambda_{1}, \lambda\right)} B_{M}^{*}(\lambda) A_{M}\left(\lambda_{1}\right) \Omega
$$

and

$$
A_{M}^{*}(\lambda) \Psi\left(\lambda_{1}\right)=\frac{1}{b\left(\lambda, \lambda_{1}\right)} B_{M}^{*}\left(\lambda_{1}\right) A_{M}(\lambda) \Omega-\frac{c\left(\lambda, \lambda_{1}\right)}{b\left(\lambda, \lambda_{1}\right)} B_{M}^{*}(\lambda) A_{M}^{*}\left(\lambda_{1}\right) \Omega .
$$

We now claim that in the general case, if $\lambda \neq \lambda_{1}, \ldots, \lambda_{N}$,

$$
\begin{aligned}
A_{M}(\lambda) \Psi\left(\lambda_{1}, \ldots, \lambda_{N}\right)= & \Lambda_{0}\left(\lambda ; \lambda_{1}, \ldots, \lambda_{N}\right) \Psi\left(\lambda_{1}, \ldots, \lambda_{N}\right) \\
& -\sum_{j=1}^{N} \Lambda_{j}\left(\lambda ; \lambda_{1}, \ldots, \lambda_{N}\right) B_{M}^{*}(\lambda) B_{M}^{*}\left(\lambda_{1}\right) \\
& \ldots B_{M}^{*}\left(\lambda_{j}\right) \ldots B_{M}^{*}\left(\lambda_{N}\right) A_{M}\left(\lambda_{j}\right) \Omega
\end{aligned}
$$


and

$$
\begin{gathered}
A_{M}^{*}(\lambda) \Psi\left(\lambda_{1}, \ldots, \lambda_{N}\right)=\tilde{\Lambda}_{0}\left(\lambda ; \lambda_{1}, \ldots, \lambda_{N}\right) \Psi\left(\lambda_{1}, \ldots, \lambda_{N}\right) \\
-\sum_{j=1}^{N} \tilde{\Lambda}_{j}\left(\lambda ; \lambda_{1}, \ldots, \lambda_{N}\right) B_{M}^{*}(\lambda) B_{M}^{*}\left(\lambda_{1}\right) \\
\ldots B_{M}^{*}\left(\lambda_{j}\right) \ldots B_{M}^{*}\left(\lambda_{N}\right) A_{M}^{*}\left(\lambda_{j}\right) \Omega,
\end{gathered}
$$

where the coefficients $\Lambda_{0}, \Lambda_{j}, \tilde{\Lambda}_{0}$ and $\tilde{\Lambda}_{j}$ are given by

$$
\begin{aligned}
& \Lambda_{0}\left(\lambda ; \lambda_{1}, \ldots, \lambda_{N}\right)=\alpha(\lambda)^{M} \prod_{j=1}^{N} \frac{1}{b\left(\lambda_{j}, \lambda\right)}, \\
& \tilde{\Lambda}_{0}\left(\lambda ; \lambda_{1}, \ldots, \lambda_{N}\right)=\bar{\alpha}(\lambda)^{M} \prod_{j=1}^{N} \frac{1}{b\left(\lambda, \lambda_{j}\right)}, \\
& \Lambda_{j}\left(\lambda ; \lambda_{1}, \ldots, \lambda_{N}\right)=\frac{c\left(\lambda_{j}, \lambda\right)}{b\left(\lambda_{j}, \lambda\right)} \prod_{i \neq j} \frac{1}{b\left(\lambda_{i}, \lambda_{j}\right)},
\end{aligned}
$$

and

$$
\tilde{\Lambda}_{j}\left(\lambda ; \lambda_{1}, \ldots, \lambda_{N}\right)=\frac{c\left(\lambda, \lambda_{j}\right)}{b\left(\lambda, \lambda_{j}\right)} \prod_{i \neq j} \frac{1}{b\left(\lambda_{j}, \lambda_{i}\right)} .
$$

To prove the formulas (4.28)-(4.31) we first remark that by symmetry

$$
\Lambda_{j}\left(\lambda ; \lambda_{1}, \ldots, \lambda_{N}\right)=\Lambda_{N}\left(\lambda ; \lambda_{1}, \ldots, \widehat{\lambda_{j}}, \ldots, \lambda_{N}, \lambda_{j}\right)
$$

and similarly for $\tilde{\Lambda}_{j}$. The induction step for the proof of (4.30) now reads

$$
\begin{aligned}
A_{M}(\lambda) & B_{M}^{*}\left(\lambda_{N+1}\right) \ldots B_{M}^{*}\left(\lambda_{1}\right) \Omega \\
= & \frac{1}{b\left(\lambda_{N+1}, \lambda\right)} B_{M}^{*}\left(\lambda_{N+1}\right) A_{M}(\lambda) B_{M}^{*}\left(\lambda_{N}\right) \ldots B_{M}^{*}\left(\lambda_{1}\right) \Omega \\
& -\frac{c\left(\lambda_{N+1}, \lambda\right)}{b\left(\lambda_{N+1}, \lambda\right)} B_{M}^{*}(\lambda) A_{M}\left(\lambda_{N+1}\right) B_{M}^{*}\left(\lambda_{N}\right) \ldots B_{M}^{*}\left(\lambda_{1}\right) \Omega \\
= & \frac{1}{b\left(\lambda_{N+1}, \lambda\right)} \Lambda_{0}\left(\lambda ; \lambda_{1}, \ldots \lambda_{N}\right) B_{M}^{*}\left(\lambda_{N+1}\right) \ldots B_{M}^{*}\left(\lambda_{1}\right) \Omega \\
& -\frac{1}{b\left(\lambda_{N+1}, \lambda\right)} \sum_{j=1}^{N} \Lambda_{j}\left(\lambda ; \lambda_{1}, \ldots, \lambda_{N}\right) \\
& -\frac{c\left(\lambda_{N+1}, \lambda\right)}{b\left(\lambda_{N+1} \lambda\right)} \Lambda_{0}\left(\lambda_{N+1} ; \lambda_{1}, \ldots, \lambda_{N}\right) B_{M}^{*}(\lambda) B_{M}^{*}\left(\lambda_{N}\right) \ldots B_{M}^{*}\left(\lambda_{1}\right) \Omega \\
& +\frac{c\left(\lambda_{N+1}, \lambda\right)}{b\left(\lambda_{N+1}, \lambda\right)} \sum_{j=1}^{N} \Lambda_{j}^{*}\left(\lambda_{N+1} ; \lambda_{1}, \ldots, \lambda_{N}\right) \\
& B_{M}^{*}(\lambda) B_{M}^{*}\left(\lambda_{N+1}\right) \ldots B_{M}^{*}\left(\lambda_{j}\right) \ldots B_{M}^{*}\left(\lambda_{1}\right) A_{M}\left(\lambda_{j}\right) \Omega .
\end{aligned}
$$


It follows in particular that

$$
\Lambda_{N+1}\left(\lambda ; \lambda_{1}, \ldots, \lambda_{N}\right)=\frac{c\left(\lambda_{N+1}, \lambda\right)}{b\left(\lambda_{N+1}, \lambda\right)} \Lambda_{0}\left(\lambda_{N+1} ; \lambda_{1} \ldots, \lambda_{N}\right)
$$

and by the symmetry relation (4.32) we obtain (4.30) with $N$ replaced by $N+1$. Now, adding (4.26) and (4.27) we conclude that $\Psi\left(\lambda_{1}, \ldots, \lambda_{N}\right)$ is an eigenvector of $A_{M}(\lambda)+A_{M}^{*}(\lambda)$ with eigenvalue

$$
E\left(\lambda ; \lambda_{1}, \ldots, \lambda_{N}\right)=\Lambda_{0}\left(\lambda ; \lambda_{1}, \ldots, \lambda_{N}\right)+\tilde{\Lambda}_{0}\left(\lambda ; \lambda_{1}, \ldots, \lambda_{N}\right)
$$

provided that $\lambda_{1}, \ldots, \lambda_{N}$ satisfy the following relations:

$$
\Lambda_{j}\left(\lambda ; \lambda_{1}, \ldots, \lambda_{N}\right) \alpha\left(\lambda_{j}\right)^{M}+\tilde{\Lambda}_{j}\left(\lambda ; \lambda_{1}, \ldots, \lambda_{N}\right) \bar{\alpha}\left(\lambda_{j}\right)^{M}=0 \quad \text { for } j=1, \ldots, N
$$

or equivalently,

$$
\left(\frac{\alpha\left(\lambda_{k}\right)}{\bar{\alpha}\left(\lambda_{k}\right)}\right)^{M}=\prod_{l \neq k} \frac{b\left(\lambda_{l}, \lambda_{k}\right)}{b\left(\lambda_{k}, \lambda_{l}\right)}
$$

for $k=1, \ldots, N$, independently of $\lambda$. These relations are just (4.12). This proves the theorem in case $\lambda \neq \lambda_{j}$. If $\lambda=\lambda_{j}$ the result follows by continuity. Indeed, $\left(A_{M}(\lambda)+A_{M}^{*}(\lambda)\right) \Psi\left(\lambda_{1}, \ldots, \lambda_{N}\right)$ is easily seen to be continuous in $\lambda$ while the relations (4.37) imply that $E_{M}\left(\lambda ; \lambda_{1}, \ldots, \lambda_{N}\right)$ has a removable singularity at $\lambda=\lambda_{j}$.

We shall need two properties of the solutions to (4.12) and the corresponding eigenfunctions (4.13). First we can take logarithms to rewrite (4.12) in a form analogous to (3.2) and (3.3):

$$
M \arctan \left(\frac{1}{2} \lambda_{k} \Delta\right)=\pi m_{k}-\sum_{l=1}^{N} \arctan \frac{\lambda_{k}-\lambda_{l}}{\kappa}
$$

if $N$ is odd, and

$$
M \arctan \left(\frac{1}{2} \lambda_{k} \Delta\right)=\pi\left(m_{k}+\frac{1}{2}\right)-\sum_{l=1}^{N} \arctan \frac{\lambda_{k}-\lambda_{l}}{\kappa}
$$

if $N$ is even. Hence we have the analogue of Lemma 3.2:

Lemma 4.1. For every set of integers $-\frac{M}{2}<m_{1}<\cdots<m_{N}<\frac{M}{2}$ there exists a unique solution $\lambda_{1}<\cdots<\lambda_{N}$ to Eqs. (4.38) respectively (4.39). Moreover, as $M \rightarrow \infty$ this solution tends to the solution of (3.2) respectively (3.3): $\lim _{M \rightarrow \infty} \lambda_{j}(M)=k_{j}$.

Proof. Analogous to (3.8) we define

$$
B_{M}\left(\lambda_{1}, \ldots, \lambda_{N}\right)=2 M \sum_{k=1}^{N} \int_{0}^{\lambda_{k}} \arctan \frac{\Delta x}{2} d x-2 \pi \sum_{k=1}^{N} I_{k} \lambda_{k}+\frac{1}{2} \sum_{k, l=1}^{N} \Theta_{\kappa}\left(\lambda_{\kappa}-\lambda_{l}\right) .
$$

It is clear that $B_{M}$ is also strictly convex and that $\partial B_{M} / \partial \lambda_{k}=0$ if and only if (4.38) respectively (4.39) holds. Furthermore,

$$
B_{M}\left(\lambda_{1}, \ldots, \lambda_{N}\right) \geqq \sum_{k=1}^{N}\left\{2 M \int_{0}^{\lambda_{k}} \arctan \frac{\Delta x}{2} d x-2 \pi I_{k} \lambda_{k}\right\} .
$$


The right-hand expression tends to infinity when $\left|\lambda_{k}\right|$ tends to infinity for some $k$ provided that $-\frac{M}{2}<I_{k}<\frac{M}{2}$. It follows that (4.38) respectively (4.39) has a unique solution in that case. To show that these solutions tend to the solutions of (3.2) respectively (3.3) we remark that $B_{M}\left(\lambda_{1}, \ldots, \lambda_{N}\right)$ converges to $B\left(\lambda_{1}, \ldots, \lambda_{N}\right)$ uniformly on compacta. This, together with strict convexity of $B$ implies that, for fixed $m_{1}, \ldots, m_{N}$ the minimiser of $B_{M}$ tends to that of $B$.

The second important property of the eigenvectors for finite $M$ is their orthogonality:

Lemma 4.2. Suppose that $\lambda_{1}<\cdots<\lambda_{N}$ and $\lambda_{1}^{\prime}<\cdots<\lambda_{N}^{\prime}$ are two different solutions of Eqs. (4.12). Then the corresponding wave functions $\Psi\left(\lambda_{1}, \ldots, \lambda_{N}\right)$ and $\Psi\left(\lambda_{1}^{\prime}, \ldots, \lambda_{N}^{\prime}\right)$ given by $(4.13)$ are orthogonal.

Proof. We shall prove that, for some $\lambda \in \mathbb{R}$,

$$
E_{M}\left(\lambda ; \lambda_{1}, \ldots, \lambda_{N}\right) \neq E_{M}\left(\lambda ; \lambda_{1}^{\prime}, \ldots, \lambda_{N}^{\prime}\right) .
$$

To this end define the functions

$$
f(\mu)=\prod_{k=1}^{N}\left(\mu-\lambda_{k}\right)
$$

and similarly

$$
g(\mu)=\prod_{k=1}^{N}\left(\mu-\lambda_{k}^{\prime}\right)
$$

for $\mu \in \mathbb{C}$, and let $W(\mu)$ be the Wronskian

$$
W(\mu)=f(\mu+i \kappa) g(\mu)-f(\mu) g(\mu+i \kappa) .
$$

Then we have by (4.14),

$$
f(\lambda) E_{M}(\lambda)=\alpha(\lambda)^{M} f(\lambda+i \kappa)+\bar{\alpha}(\lambda)^{M} f(\lambda-i \kappa)
$$

and similarly for $g$. Assuming that $E_{M}(\lambda)=E_{M}^{\prime}(\lambda)$ for all real $\lambda$ we obtain

$$
\begin{aligned}
W(\lambda-i \kappa)= & f(\lambda) g(\lambda-i \kappa)-f(\lambda-i \kappa) g(\lambda) \\
= & f(\lambda)\left\{\frac{E_{M}(\lambda)}{(\bar{\lambda})^{M}} g(\lambda)-\left(\frac{\alpha(\lambda)}{\bar{\alpha}(\lambda)}\right)^{M} g(\lambda+i \kappa)\right\} \\
& -g(\lambda)\left\{\frac{E_{M}(\lambda)}{\bar{\alpha}(\lambda)^{M}} f(\lambda)-\left(\frac{\alpha(\lambda)}{\bar{\alpha}(\lambda)}\right)^{M} f(\lambda+i \kappa)\right\} \\
= & \left(\frac{\alpha(\lambda)}{\bar{\alpha}(\lambda)}\right)^{M} W(\lambda) .
\end{aligned}
$$

On the other hand

$$
W(\lambda-i \kappa)=i \kappa\left(\prod_{k=1}^{N}\left(\lambda-\lambda_{k}^{\prime}\right)-\prod_{k=1}^{N}\left(\lambda-\lambda_{k}\right)\right)=W(\lambda) .
$$

It follows that $W(\lambda)=0$ except for a finite number of $\lambda$ 's. By continuity, $W(\lambda)=0$ for all real $\lambda$. By (4.46) this implies that $\left\{\lambda_{k}\right\}=\left\{\lambda_{k}^{\prime}\right\}$. 


\section{Convergence of the Lattice Approximation}

To prove the orthogonality of the Bethe Ansatz eigenstates it now suffices to prove that the eigenstates (4.13) of the lattice model converge to the Bethe Ansatz eigenstates as $\Delta \rightarrow 0$. We first embed the Fock space $\mathscr{F}^{\Delta}$ into the continuum Fock space $\mathscr{F}=\bigoplus_{N=0}^{\infty} L_{\text {sym }}^{2}\left([0,1]^{N}\right)$ by the mapping

$$
\begin{aligned}
& \sum_{1 \leqq n_{1} \leqq} f\left(n_{1}, \ldots, n_{N}\right) a_{n_{1}}^{*} \cdots a_{n_{N}}^{*} \Omega \mapsto
\end{aligned}
$$

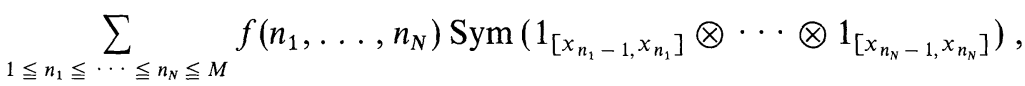

where the symmetrisation operator Sym is defined by (2.5). It is easily seen that this mapping is an isometry for all $M$. Indeed.

$$
\begin{aligned}
\| \operatorname{Sym} & \left(1_{\left[x_{n_{1}}-1, x_{n_{1}}\right]} \otimes \cdots \otimes 1_{\left[x_{n_{N}-1}, x_{n_{N}}\right]}\right) \|^{2} \\
& =\sum_{\sigma \in \mathscr{S}_{N}} \prod_{i=1}^{N} \int_{x_{n_{i}-1}}^{x_{n_{i}}} d x_{i} 1_{\left[x_{n_{\sigma(i)}-1}, x_{\left.n_{\sigma(i)}\right]}\left(x_{i}\right)=\theta\left(n_{1}, \ldots, n_{N}\right) .\right.}
\end{aligned}
$$

Next we rewrite the Bethe Ansatz eigenstates in a more symmetrical way introduced by Gaudin (see [19]):

Lemma 5.1. The Bethe Ansatz eigenstate $\psi_{\left\{k_{j}\right\}}^{B A}$ given by (3.1) where the conditions (3.2)-(3.6) are satisfied can be written as follows:

$$
\psi_{\left\{k_{j}\right\}}^{B A}\left(x_{1}, \ldots, x_{N}\right)=\sqrt{N !} \operatorname{Sym}\left(\tilde{\psi}_{\left\{k_{j}\right\}}\right)\left(x_{1}, \ldots, x_{N}\right),
$$

where

$$
\tilde{\psi}_{\left\{k_{j}\right\}}\left(x_{1}, \ldots, x_{N}\right)=\prod_{1 \leqq i<j \leqq N}\left(1-\frac{i \kappa}{k_{i}-k_{j}} \varepsilon\left(x_{i}-x_{j}\right)\right) \prod_{j=1}^{N} e^{i k_{j} x_{j}} .
$$

Proof. Let $x_{1}<\cdots<x_{N}$. We shall prove that the coefficients of $\exp \left[i \sum_{j=1}^{N} k_{\sigma(j)} x_{j}\right]$ satisfy (3.5) when $\sigma$ and $\tau$ differ by a single transposition as in (3.6). Now, assuming that $k_{1}<\cdots<k_{N}$,

$$
\sqrt{N !} \operatorname{Sym} \tilde{\psi}\left(x_{1}, \ldots, x_{N}\right)=\sum_{\sigma \in \mathscr{S}_{N}} A_{\sigma}\left(k_{1}, \ldots, k_{N}\right) \prod_{j=1}^{N} e^{\imath k_{\sigma(j)} x_{j}},
$$

where

$$
A_{\sigma}\left(k_{1}, \ldots, k_{N}\right)=\prod_{(l, j): \sigma(l)<\sigma(J)}\left(1-\frac{i \kappa}{k_{\sigma(i)}-k_{\sigma(j)}} \varepsilon\left(k_{i}-k_{j}\right)\right) .
$$

If $\sigma$ and $\tau$ differ by a transposition as in (3.6) then $\sigma(i)<\sigma\left(i^{\prime}\right) \Leftrightarrow \tau(i)<\tau\left(i^{\prime}\right)$ when $i, i^{\prime} \neq j, j+1$, while $\sigma(j)<\sigma(i) \Leftrightarrow \tau(j+1)<\tau(i)$ if $i \neq j+1$ and $\sigma(j+1)<\sigma(i) \Leftrightarrow \tau(j)<\tau(i)$ if $i \neq j$. It follows that

$$
\frac{A_{\tau}}{A_{\sigma}}=\frac{1 \mp \frac{i \kappa}{k_{\tau(j+1)}-k_{\tau(j)}}}{1 \pm \frac{i \kappa}{k_{\sigma(j)}-k_{\sigma(j+1)}}}=-\exp \left[-i \theta_{\kappa}\left(k_{\tau(j)}-k_{\sigma(j)}\right)\right]
$$


The proof of the convergence of the lattice approximation will now be subdivided into two main lemmas: Lemma 5.2 and Lemma 5.4. The first contains the "analytical" part of the proof and the second the "algebraic" part in a sense. Indeed, to reduce the problem to an algebraic one we must show that the additional terms in the monodromy matrices for the lattice model are negligible in the limit $\Delta \rightarrow 0$ compared with the terms originally considered by Faddeev et al. $[16,17,31]$. To be precise we prove:

Lemma 5.2. In the limit $\Delta \rightarrow 0$, the eigenfunction $\Psi\left(\lambda_{1}, \ldots, \lambda_{N}\right)$ defined in (4.13) is given by

where

$$
\Psi\left(\lambda_{1}, \ldots, \lambda_{N}\right) \approx\left(\prod_{k=1}^{N}\left(\tilde{B}_{0}^{*}\left(\lambda_{k}\right)+\cdots+\tilde{B}_{k-1}^{*}\left(\lambda_{k}\right)\right)\right) \Omega+O(\Delta),
$$

$$
\tilde{B}_{j}^{*}(\lambda)=\sum_{1 \leqq n_{1}<\cdots<n_{j} \leqq M} \beta_{M, n_{j}+1}^{*}(\lambda) \beta_{n_{J}} \beta_{n_{\jmath}-1, n_{j}-1}^{*}(\lambda) \ldots \beta_{n_{2}-1, n_{1}+1}^{*} \beta_{n_{1}} \beta_{n_{1}-1,1}^{*}
$$

and

$$
\beta_{m, n}^{*}(\lambda)=\sum_{r=n}^{m} \alpha(\lambda)^{m-r} \bar{\alpha}(\lambda)^{r-n} \beta_{r}^{*}
$$

Moreover, the operators $B_{M}^{*}(\lambda)$ defined by (4.9) are bounded on $\mathscr{F}_{N}^{\Delta}$ by a constant independent of $M$ (but depending on $N$ ).

Remark. In the following we shall omit the $O(\Delta)$ symbol and simply write the $\approx$ sign to indicate equality up to order $\Delta$.

Proof. We prove both statements in the lemma simultaneously by induction on $N$. Consider first the case $N=1$. We write

where

$$
L_{n}(\lambda)=L_{n}^{0}(\lambda)+\tilde{L}_{n}
$$

$$
L_{n}^{0}(\lambda)=\left(\begin{array}{cc}
\alpha(\lambda) & \beta_{n}^{*} \\
0 & \bar{\alpha}(\lambda)
\end{array}\right)
$$

and

$$
\tilde{L}_{n}=\left(\begin{array}{cc}
\frac{1}{2} \kappa a_{n}^{*} a_{n} & 0 \\
\beta_{n} & \frac{1}{2} \kappa a_{n}^{*} a_{n}
\end{array}\right) .
$$

(In (5.12) we have written $\alpha(\lambda)$ instead of $\alpha(\lambda) 1$ with a slight abuse of notation; $\alpha(\lambda)$ is given by (4.11).) Then

$$
L_{M}\left(\lambda_{1}\right) \ldots L_{1}\left(\lambda_{1}\right) \Omega=L_{M}^{0}\left(\lambda_{1}\right) \ldots L_{1}^{0}\left(\lambda_{1}\right) \Omega=\left(\begin{array}{cc}
\alpha\left(\lambda_{1}\right)^{M} & \beta_{M, 1}^{*}\left(\lambda_{1}\right) \\
0 & \bar{\alpha}\left(\lambda_{1}\right)^{M}
\end{array}\right) \Omega
$$

(We define in general, $\left(\begin{array}{cc}A & C \\ B & D\end{array}\right) \Psi=\left(\begin{array}{cc}A \Psi & C \Psi \\ B \Psi & D \Psi\end{array}\right)$.) It follows that

$$
\Psi\left(\lambda_{1}\right)=B_{M}^{*}\left(\lambda_{1}\right) \Omega=\beta_{M, 1}^{*}\left(\lambda_{1}\right) \Omega=\widetilde{B}_{0}^{*}\left(\lambda_{1}\right) \Omega .
$$


For general $N$ and $\Psi \in \mathscr{F}_{N}^{A}$ the decomposition (5.11) leads to

$$
\begin{aligned}
& L_{M}(\lambda) \ldots L_{1}(\lambda) \Psi \\
& =L_{M}^{0}(\lambda) \ldots L_{1}^{0}(\lambda) \Omega \\
& \quad+\sum_{n_{1}=1}^{M} L_{M, n_{1}+1}^{0}(\lambda) \tilde{L}_{n_{1}} L_{n_{1}-1,1}(\lambda) \Psi+\cdots \\
& \quad+\sum_{1 \leqq n_{1}<\cdots<n_{N} \leqq M} L_{M, n_{N}+1}^{0}(\lambda) \tilde{L}_{n_{N}} L_{n_{N}-1, n_{N-1}+1}^{0}(\lambda) \tilde{L}_{n_{N}-1} \ldots \tilde{L}_{n_{1}} L_{n_{1}-1,1}^{0}(\lambda) \Psi .
\end{aligned}
$$

Here,

$$
L_{m, n}^{0}(\lambda)=\prod_{k=n}^{m} L_{k}^{0}(\lambda) .
$$

In the proof of $(5.8)$ we shall show that

$$
\begin{gathered}
\left(\sum_{1 \leqq n_{1}<} \sum_{<n_{p} \leqq M} L_{M, n_{p}+1}^{0}(\lambda) \tilde{L}_{n_{p}} \ldots \tilde{L}_{n_{1}} L_{n_{1}-1,1}^{0}(\lambda)\right)_{(1,2)} \Psi\left(\left(\lambda_{1}, \ldots, \lambda_{N}\right)\right. \\
\approx \tilde{B}_{p}^{*}(\lambda) \Psi\left(\lambda_{1}, \ldots, \lambda_{N}\right) .
\end{gathered}
$$

To prove that $B_{M}^{*}(\lambda)$ is bounded on $\mathscr{F}_{N}^{\Delta}$ it is sufficient to show that the $(1,2)$ Ycomponent (5.17) of each term in (5.15) is bounded by a constant times $\|\Psi\|$. The case $p=0$ follows immediately from (5.14): it equals $\beta_{M, 1}^{*}(\lambda) \Psi$. To bound this we write $\Psi$ in the form (4.2). Then, using (5.10),

$$
\begin{aligned}
\beta_{M, 1}^{*}(\lambda) \Psi \\
=-i \sqrt{\kappa} \sum_{n=1}^{M} \sum_{1 \leqq n_{1} \leqq \cdots \leqq n_{N} \leqq M} f\left(n_{1}, \ldots, n_{N}\right) \alpha(\lambda)^{M-n} \bar{\alpha}(\lambda)^{n-1} a_{n}^{*} \rho_{n} a_{n_{1}}^{*} \ldots a_{n_{N}}^{*} \Omega \\
=-i \sqrt{\kappa} \sum_{1 \leqq n_{1} \leqq} \sum_{\leqq n_{N+1} \leqq M} \sum_{j=1}^{N+1} \frac{f\left(n_{1}, \ldots, \widehat{n_{j}}, \ldots, n_{N+1}\right)}{1+\#\left\{i \neq j \mid n_{i}=n_{j}\right\}} \alpha(\lambda)^{M-n_{j}} \bar{\alpha}(\lambda)^{n_{j}-1} \\
\quad \times\left(1+\frac{1}{4} \kappa \Delta \ddagger\left\{i \neq j \mid n_{i}=n_{j}\right\}\right)^{1 / 2} a_{n_{1}}^{*} \ldots a_{n_{N+1}}^{*} \Omega .
\end{aligned}
$$

Hence

$$
\begin{aligned}
\left\|\beta_{M, 1}^{*} \Psi\right\|^{2} \leqq & \kappa \Delta^{N+1}\left(1+\frac{1}{4} \kappa \Delta N\right)_{1 \leqq n_{1} \leqq \cdots \leqq n_{N+1} \leqq M} \theta\left(n_{1}, \ldots, n_{N+1}\right) \\
& \times\left|\sum_{j=1}^{N+1} \frac{f\left(n_{1}, \ldots, \widehat{n_{j}}, \ldots, n_{N+1}\right)}{1+\#\left\{i \neq j \mid n_{i}=n_{j}\right\}} \alpha(\lambda)^{M-n_{j}} \bar{\alpha}(\lambda)^{n_{j}-1}\right|^{2} .
\end{aligned}
$$

But, by Cauchy-Schwarz,

$$
\begin{aligned}
& \left|\sum_{j=1}^{N+1} \frac{f\left(n_{1}, \ldots, \widehat{n_{j}}, \ldots, n_{N+1}\right)}{1+\#\left\{i \neq j \mid n_{i}=n_{j}\right\}} \alpha(\lambda)^{M-n_{j}} \bar{\alpha}(\lambda)^{n_{j}-1}\right|^{2} \\
& \quad \leqq \sum_{j=1}^{N+1} \frac{\left|f\left(n_{1}, \ldots, \widehat{n_{j}}, \ldots, n_{N+1}\right)\right|^{2}}{1+\#\left\{i \neq j \mid n_{i}=n_{j}\right\}} \cdot \sum_{j=1}^{N+1}\left|\alpha(\lambda)^{M-n_{j}} \bar{\alpha}(\lambda)^{n_{j}-1}\right|^{2} \\
& \quad \leqq(N+1)\left(1+\lambda^{2} \Delta^{2} / 4\right)^{M-1} \sum_{j=1}^{N+1} \frac{\left|f\left(n_{1}, \ldots, \widehat{n}_{j}, \ldots, n_{N+1}\right)\right|^{2}}{1+\#\left\{i \neq j \mid n_{i}=n_{j}\right\}} .
\end{aligned}
$$


Using the fact that $\theta\left(n_{1}, \ldots, n_{N+1}\right) \leqq(N+1) \theta\left(n_{1}, \ldots, \widehat{n_{j}}, \ldots, n_{N+1}\right)$ we conclude that

$$
\begin{aligned}
\left\|\beta_{M, 1}^{*}(\lambda) \Psi\right\|^{2} \leqq & \kappa \Delta^{N+1}\left(1+\frac{1}{4} \kappa \Delta N\right)(N+1)\left(1+\frac{1}{4} \lambda^{2} \Delta^{2}\right)^{M} \\
& \times \sum_{1 \leqq n_{1} \leqq \cdots \leqq n_{N+1} \leqq M} \sum_{j=1}^{N+1} \frac{\left|f\left(n_{1}, \ldots, \widehat{n_{j}}, \ldots, n_{N+1}\right)\right|^{2}}{1+\mp\left\{i \neq j \mid n_{i}=n_{j}\right\}} \\
\leqq & \kappa \Delta^{N+1}(N+1)^{2}(1+\kappa \Delta N / 4)\left(1+n_{1}, \ldots, n_{N+1}\right) \\
& \times \sum_{n=1}^{M} \Delta_{1 \leqq n_{1} \leqq \cdots \leqq n_{N} \leqq M}\left|f\left(n_{1}, \ldots, n_{N}\right)\right|^{2} \theta\left(n_{1}, \ldots, n_{N}\right) \\
= & \kappa\|\Psi\|^{2}(N+1)^{2} K_{M}(\lambda)^{2} \gamma_{M}^{2},
\end{aligned}
$$

where

$$
K_{M}(\lambda)=\left(1+\lambda^{2} \Delta^{2} / 4\right)^{M / 2} \quad \text { and } \quad \gamma_{M}=(1+\kappa N \Delta / 4)^{1 / 2}
$$

Since $\lim \sup _{M \rightarrow \infty} K_{M}(\lambda)=1$ and $\lim \sup _{M \rightarrow \infty} \gamma_{M}=1$, this implies that $\beta_{M, 1}^{*}(\lambda)$ is bounded uniformly in $M$. Indeed,

$$
\limsup _{M \rightarrow \infty}\left\|\beta_{M, 1}^{*}(\lambda)\right\|_{\mathscr{F}_{N}} \leqq \sqrt{\kappa}(N+1) .
$$

Let us now consider the $p^{\text {th }}$ term in the series (5.15). To this end define for general $p \leqq m \leqq M$ and $\Psi \in \mathscr{F}_{N}^{\Delta}$ :

$$
\Gamma_{m}^{(p)}=\sum_{1 \leqq n_{1}<\cdots<n_{p} \leqq m} L_{m, n_{p}+1}^{0}(\lambda) \tilde{L}_{n_{p}} \ldots \tilde{L}_{n_{1}} L_{n_{1}-1,1}^{0}(\lambda) \Psi
$$

We then have

$$
\Gamma_{m}^{(p)}=\sum_{n=1}^{m} L_{m, n+1}^{0}(\lambda) \tilde{L}_{n} \Gamma_{n-1}^{(p-1)}
$$

In particular, if $\underline{\Psi}_{m}^{(p)}$ is the second column of $\Gamma_{m}^{(p)}$ then $\left(\underline{\Psi}_{M}^{(p)}\right)_{1}$ is just the left-hand side of (5.17), and by (5.24),

$$
\begin{aligned}
\left(\underline{\Psi}_{m}^{(p)}\right)_{1}= & \sum_{n=1}^{m}\left(\frac{1}{2} \kappa \alpha(\lambda)^{m-n} a_{n}^{*} a_{n}+\beta_{m, n+1}^{*} \beta_{n}\right)\left(\underline{\Psi}_{n-1}^{(p-1)}\right)_{1} \\
& +\frac{1}{2} \kappa \sum_{n=1}^{m} \beta_{m, n+1}^{*} a_{n}^{*} a_{n}\left(\underline{\Psi}_{n-1}^{(p-1)}\right)_{2}
\end{aligned}
$$

and

$$
\left(\underline{\Psi}_{m}^{(p)}\right)_{2}=\sum_{n=1}^{m} \bar{\alpha}(\lambda)^{m-n} \beta_{n}\left(\underline{\Psi}_{n-1}^{(p-1)}\right)_{1}+\frac{1}{2} \kappa \sum_{n=1}^{m} \bar{\alpha}(\lambda)^{m-n} a_{n}^{*} a_{n}\left(\underline{\Psi}_{n-1}^{(p-1)}\right)_{2} .
$$

For the first term in (5.25) we have

$$
\frac{1}{2} \kappa|\alpha(\lambda)|^{m-1}\left\|\sum_{n=1}^{m} a_{n}^{*} a_{n}\left(\underline{\Psi}_{n-1}^{(p-1)}\right)_{1}\right\| \leqq \frac{1}{2} \kappa K_{m}(\lambda) \Delta(N+1) \sup _{p \leqq n \leqq M}\left\|\left(\underline{\Psi}_{n-1}^{(p-1)}\right)_{1}\right\|
$$


because $\left(\underline{\Psi}_{m}^{(p)}\right)_{1} \in \mathscr{F}_{N+1}^{\Delta}$. This term is therefore of order $\Delta$. Similarly, the second term in $(5.26)$ is of order $\Delta$. In the remaining terms we write $\left(\underline{\Psi}_{n-1}^{(p-1)}\right)_{1}$ and $\left(\underline{\Psi}_{n-1}^{(p-1)}\right)_{2}$ in the form (4.2):

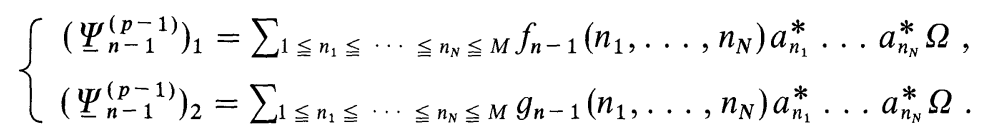

The last term of (5.25) can then be bounded analogous to (5.19):

$$
\begin{aligned}
& \left\|\sum_{n=1}^{m} \beta_{m, n+1}^{*}(\lambda) a_{n}^{*} a_{n}\left(\underline{\Psi}_{n-1}^{(p-1)}\right)_{2}\right\|^{2} \\
& \leqq \kappa \Delta^{N+3} \gamma_{M}^{2} \sum_{1 \leqq n_{1} \leqq} \sum_{n_{N+1} \leqq M} \theta\left(n_{1}, \ldots, n_{N+1}\right) \\
& \times\left|\sum_{j=1}^{N+1} \chi\left(n_{j} \leqq m\right) \sum_{i=1}^{j-1} \frac{g_{n_{i}-1}\left(n_{1}, \ldots, \widehat{n_{j}}, \ldots, n_{N+1}\right)}{1+\#\left\{k \neq j \mid n_{k}=n_{j}\right\}} \alpha(\lambda)^{m-n_{j}} \bar{\alpha}(\lambda)^{n_{j}-1}\right|^{2} \\
& \leqq \kappa \Delta^{2} N^{2}(N+1)^{2} K_{M}(\lambda)^{2} \gamma_{M}^{2} \sup _{p \leqq n \leqq M}\left\|\left(\underline{\Psi}_{n-1}^{(p-1)}\right)_{2}\right\|^{2} .
\end{aligned}
$$

The last term of (5.25) is therefore also of order $\Delta$. We conclude that

$$
\left(\underline{\Psi}_{m}^{(p)}\right)_{1} \approx \sum_{n=1}^{m} \beta_{m, n+1}^{*}(\lambda) \beta_{n}\left(\underline{\Psi}_{n-1}^{(p-1)}\right)_{1}+O(\Delta)
$$

uniformly in $m$ provided that the remaining terms in (5.25) and (5.26) can be bounded uniformly in $m$. Formula (5.30) of course leads to (5.9).

We bound the first term of (5.26) as follows:

$$
\begin{aligned}
\sum_{n=1}^{m} \bar{\alpha}(\lambda)^{m-n} \beta_{n}\left(\underline{\Psi}_{n-1}^{(p-1)}\right)_{1} \\
=i \sqrt{\kappa} \Delta \sum_{1 \leqq n_{1} \leqq \cdots \leqq n_{N} \leqq M} \sum_{n=1}^{m} \#\left\{i \mid n_{i}=n\right\} \bar{\alpha}(\lambda)^{m-n} \tilde{\rho}_{n} f_{n-1}\left(n_{1}, \ldots, n_{N}, n\right) \\
\quad \times a_{n_{1}}^{*} \ldots a_{n_{N}}^{*} \Omega .
\end{aligned}
$$

Here $\tilde{\rho}_{n}=\rho_{n}\left(n_{1}, \ldots, n_{N}\right)=\left(1+\frac{1}{4} \kappa \Delta \#\left\{i \mid n_{i}=n\right\}\right)^{1 / 2}$. Hence

$$
\begin{aligned}
\left\|\sum_{n=1}^{m} \bar{\alpha}(\lambda)^{m-n} \beta_{n}\left(\underline{\Psi}_{n-1}^{(p-1)}\right)_{1}\right\|^{2} & \\
\leqq & \kappa \gamma_{M} \Delta^{N+2} \sum_{1 \leqq n_{1} \leqq \cdots n_{N} \leqq M}\left(\sum_{n=1}^{m}\left(\sharp\left\{i \mid n_{i}=n\right\}\right)^{2}|\bar{\alpha}(\lambda)|^{2(m-n)}\right) \\
& \quad \times\left(\sum_{n=1}^{m}\left|f_{n-1}\left(n_{1}, \ldots, n_{N}, n\right)\right|^{2}\right) \theta\left(n_{1}, \ldots, n_{N}\right) \\
\leqq & \kappa \gamma_{M} \Delta^{N+2} K_{m}(\lambda)^{2} N^{2} \sum_{1 \leqq n_{1} \leqq} \sum_{n_{N} \leqq M} \\
& \times \sum_{n=1}^{m}\left|f_{n-1}\left(n_{1}, \ldots, n_{N}, n\right)\right|^{2} \theta\left(n_{1}, \ldots, n_{N}\right) \\
\leqq & \kappa \gamma_{M} \Delta K_{M}(\lambda)^{2}(N+1) N^{2} \sup _{p \leqq n \leqq m}\left\|\underline{\Psi}_{n-1}^{(p-1)}\right\|^{2} .
\end{aligned}
$$


The first term of (5.25) can be written as follows:

$$
\begin{aligned}
& \sum_{n=1}^{m} \beta_{M, n+1}^{*}(\lambda) \beta_{n}\left(\underline{\Psi}_{n-1}^{(p-1)}\right)_{1} \\
& =\kappa \Delta \sum_{1 \leqq n_{1} \leqq} \sum_{k=2} \sum_{n_{N} \leqq M}^{M} \tilde{\rho}_{k} \alpha(\lambda)^{M-k} \bar{\alpha}(\lambda)^{k-1} \\
& \times\left\{\sum_{n=1}^{k-1} \bar{\alpha}(\lambda)^{-n} \tilde{\rho}_{n}\left(1+\#\left\{i \mid n_{i}=n\right\}\right) f\left(n_{1}, \ldots, n_{N}, n\right)\right\} a_{k}^{*} a_{n_{1}}^{*} \ldots a_{n_{N}}^{*} \Omega \\
& =\kappa \Delta \sum_{1 \leqq n_{1} \leqq} \sum_{j=1}^{N+1}\left(1-\delta_{n_{j}, 1}\right) \tilde{\rho}_{n_{j}} \frac{\alpha(\lambda)^{M-n_{j}} \bar{\alpha}(\lambda)^{n_{j}-1}}{1+\#\left\{i \neq j \mid n_{i}=n_{j}\right\}} \\
& \times\left\{\sum_{n=1}^{n_{j}-1} \bar{\alpha}(\lambda)^{-n} \tilde{\rho}_{n}\left(1+\#\left\{i \mid n_{i}=n\right\}\right) f\left(n_{1}, \ldots, \widehat{n_{j}}, \ldots, n_{N+1}, n\right)\right\} \\
& \times a_{n_{1}}^{*} \ldots a_{n_{N+1}}^{*} \Omega \text {. }
\end{aligned}
$$

This leads to the following bound on the norm:

$$
\begin{aligned}
& \left\|\sum_{n=1}^{m} \beta_{M, n+1}^{*}(\lambda) \beta_{n}\left(\underline{\Psi}_{n-1}^{(p-1)}\right)_{1}\right\|^{2} \\
& \leqq \\
& \quad \kappa^{2} \Delta^{N+3} \gamma_{M}^{4}(N+1)^{3} K_{M}(\lambda)^{2} \\
& \quad \times\left.\left.\quad \sum_{1 \leqq n_{1} \leqq} \sum_{j=1}^{N+1}\right|_{n=1} ^{n_{j}-1} f_{n-1}\left(n_{1}, \ldots, \widehat{n}_{j}, \ldots, n_{N+1}, n\right)\right|^{2} \\
& \quad \times \theta\left(n_{1}, \ldots, n_{N+1}\right) \\
& \leqq \\
& \kappa^{2} \gamma_{M}^{4}(N+1)^{4} K_{M}(\lambda)^{2(M-1)} \sup _{p \leqq n \leqq M}\left\|\left(\underline{\Psi}_{n-1}^{(p-1)}\right)_{1}\right\|^{2} .
\end{aligned}
$$

This concludes the proof of Lemma 5.2.

The algebraic part of the proof also uses induction. The induction step makes use of

Lemma 5.3. Let $C_{\sigma}\left(\lambda_{1}, \ldots, \lambda_{N}\right)$ be defined by

$$
C_{\sigma}\left(\lambda_{1}, \ldots, \lambda_{N}\right)=\prod_{(i, j) . \tilde{\sigma}(i)<\bar{\sigma}(j)}\left(1-\frac{\kappa}{i\left(\lambda_{\bar{\sigma}(i)}-\lambda_{\bar{\sigma}(J)}\right)} \varepsilon_{i j}\right),
$$

where $\bar{\sigma}$ is the inverse of the permutation $\sigma$ and

$$
\varepsilon_{i j}= \begin{cases}+1 & \text { if } i<j \\ -1 & \text { if } i>j\end{cases}
$$

Then, if $x_{1}<\cdots<x_{N}$,

$$
\psi_{\left\{k_{j}\right\}}^{B A}\left(x_{1}, \ldots, x_{N}\right)=\sum_{\sigma \in \mathscr{S}_{N}} C_{\sigma}\left(k_{1}, \ldots, k_{N}\right) \prod_{j=1}^{N} e^{i k_{\tilde{\sigma}(\jmath)} x_{j}}
$$


and

$$
C_{\sigma}\left(\lambda_{1}, \ldots, \lambda_{N}\right)=\prod_{\substack{i=1 \\ i \neq \sigma(N)}}^{N}\left(1-\frac{\kappa}{i\left(\lambda_{\bar{\sigma}(l)}-\lambda_{N}\right)} \varepsilon_{i, \sigma(N)}\right) C_{\sigma^{\prime}}\left(\lambda_{1}, \ldots, \lambda_{N-1}\right),
$$

where $\sigma^{\prime} \in \mathscr{S}_{N-1}$ is defined by

$$
\sigma^{\prime}(i)<\sigma^{\prime}(j) \Leftrightarrow \sigma(i)<\sigma(j) .
$$

Proof. Equation (5.36) follows from (5.3), (5.5) and (5.6). (5.37) is immediate from the definition of $C_{\sigma}$.

Lemma 5.4. For arbitrary $N$,

$$
B_{M}^{*}\left(\lambda_{N}\right) \ldots B_{M}^{*}\left(\lambda_{1}\right) \Omega \approx \sum_{1 \leqq n_{1} \leqq \cdots \leqq n_{N} \leqq M} f_{N}\left(n_{1}, \ldots, n_{N}\right) a_{n_{1}}^{*} \ldots a_{n_{N}}^{*} \Omega+O(\Delta),
$$

where

$$
f_{N}\left(n_{1}, \ldots, n_{N}\right)=(-i \sqrt{\kappa})^{N} \sum_{\sigma \in \mathscr{S}_{N}} C_{\sigma}\left(\lambda_{1}, \ldots, \lambda_{N}\right) \prod_{i=1}^{N} \alpha\left(\lambda_{i}\right)^{M-n_{\sigma(i)}} \bar{\alpha}\left(\lambda_{i}\right)^{n_{\sigma(i)}} .
$$

Proof. We use induction on $N$. For $N=1$ we have already seen that

$$
\Psi\left(\lambda_{1}\right)=\beta_{M, 1}^{*}\left(\lambda_{1}\right) \Omega=\sum_{n=1}^{M} \alpha\left(\lambda_{1}\right)^{M-n} \bar{\alpha}\left(\lambda_{1}\right)^{n-1} \beta_{n}^{*} \Omega \approx \sum_{n=1}^{M} f_{1}(n) a_{n}^{*} \Omega,
$$

where $f_{1}(n)=(-i \sqrt{\kappa}) \alpha\left(\lambda_{1}\right)^{M-n} \bar{\alpha}\left(\lambda_{1}\right)^{n}$. Notice that $\rho_{n} \Omega=\Omega$. In general we have, for $\Psi \in \mathscr{F}_{N}^{\Delta}, \rho_{n} \Psi \approx \Psi$ since $N$ is bounded. We may also neglect factors $\alpha(\lambda)$ since these are of order $(1+O(\Delta))$. In the induction step we now prove that

$$
\Psi_{k}^{(N)}:=\tilde{B}_{k}^{*}\left(\lambda_{N}\right) \Psi\left(\lambda_{1}, \ldots, \lambda_{N-1}\right) \approx \Psi_{k, 0}^{(N)}+\Psi_{k,+}^{(N)}+\Psi_{k,-}^{(N)},
$$

where

$$
\Psi_{0,-}^{(N)}=\Psi_{N-1,+}^{(N)}=0 ; \quad \Psi_{k,-}^{(N)}+\Psi_{k-1,+}^{(N)}=0
$$

and

$$
\Psi_{k, 0}^{(N)}=\sum_{1 \leqq n_{1}<\cdots<n_{N} \leqq M} f_{N}^{(k)}\left(n_{1}, \ldots, n_{N}\right) a_{n_{1}}^{*} \ldots a_{n_{N}}^{*} \Omega
$$

with

$$
f_{N}^{(k)}\left(n_{1}, \ldots, n_{N}\right)=(-i \sqrt{\kappa})^{N} \sum_{\sigma \in \mathscr{S}_{N}} C_{\sigma}^{(k)}\left(\lambda_{1}, \ldots, \lambda_{N}\right) \prod_{i=1}^{N} \alpha\left(\lambda_{i}\right)^{M-n_{\sigma(i)}} \bar{\alpha}\left(\lambda_{i}\right)^{n_{\sigma(i)}}
$$

and

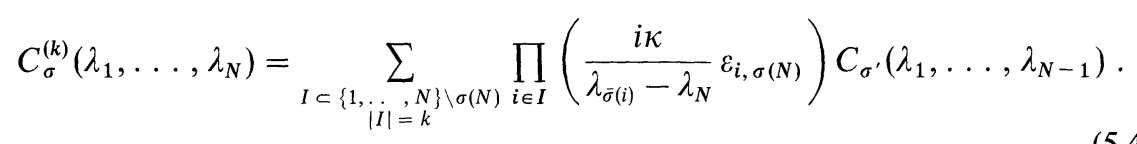

This yields (5.39) with the help of Lemma 5.3. 
The left-hand side of (5.42) can be written out as follows:

$$
\begin{aligned}
\Psi_{k}^{(N)} \approx & (-i \sqrt{\kappa})^{N} \kappa^{k} \sum_{1 \leqq n_{1}<\cdots<n_{k} \leqq M} \sum_{r_{1}=1}^{n_{1}-1} \sum_{r_{2}=n_{1}+1}^{n_{2}-1} \cdots \sum_{r_{k+1}=n_{k}+1}^{M} \\
& \alpha\left(\lambda_{N}\right)^{M-\left(r_{1}+\cdots+r_{k+1}\right)+\left(n_{1}+\cdots+n_{k}\right)-k} \\
& \times \bar{\alpha}\left(\lambda_{N}\right)^{\left(r_{1}+\cdots+r_{k+1}\right)-\left(n_{1}+\cdots+n_{k}\right)-k} \\
& \times \sum_{1 \leqq m_{1}<\cdots<m_{N-1} \leqq M} \sum_{\tau \in \mathscr{P}_{N-1}} C_{\tau}\left(\lambda_{1}, \ldots, \lambda_{N-1}\right) \\
& \times \prod_{i=1}^{N-1} \alpha\left(\lambda_{i}\right)^{M-m_{\tau(i)}} \bar{\alpha}\left(\lambda_{i}\right)^{m_{\tau(i)}} a_{r_{k+1}}^{*} a_{n_{k}} a_{r_{k}}^{*} \ldots a_{n_{1}} a_{r_{1}}^{*} a_{m_{1}}^{*} \ldots a_{m_{N-1}}^{*} \Omega .
\end{aligned}
$$

It is easy to see that the terms with $r_{i}=m_{j}$ for some $i$ and $j$ are negligible in this sum. Furthermore, for every $i=1, \ldots, k$ there must exist $j(i)$ such that $n_{i}=m_{j(i)}$. We can therefore write $\left\{r_{1}, \ldots, r_{k+1}\right\} \cup\left\{m_{1}, \ldots, m_{N-1}\right\} \backslash\left\{n_{1}, \ldots, n_{k}\right\}=$ $\left\{m_{1}^{\prime}, \ldots, m_{N}^{\prime}\right\}$, where $m_{1}^{\prime}<\cdots<m_{N}^{\prime}$ and $r_{a}=m_{i_{a}}^{\prime}$ with $1 \leqq i_{1}<\cdots<i_{k+1}$ $\leqq N$. The expression (5.47) then becomes

$$
\begin{aligned}
& \Psi_{k}^{(N)} \approx(-i \sqrt{\kappa})^{N}(\kappa \Delta)^{k} \sum_{1 \leqq m_{1}^{\prime}<\cdots<m_{N}^{\prime} \leqq M} \sum_{1 \leqq i_{1}<} \sum_{<i_{k+1} \leqq N}
\end{aligned}
$$

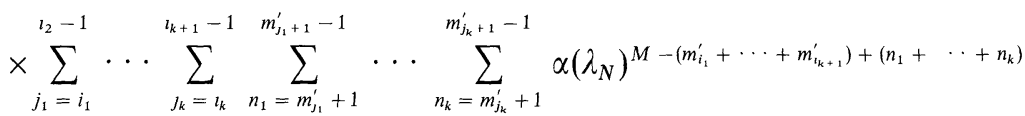

$$
\begin{aligned}
& \times \bar{\alpha}\left(\lambda_{N}\right)^{\left(m_{i_{1}}^{\prime}+\cdots+m_{i_{k+1}}^{\prime}\right)-\left(n_{1}+\cdots+n_{k}\right)} \sum_{\tau \in \mathscr{S}_{N-1}} C_{\tau}\left(\lambda_{1}, \ldots, \lambda_{N-1}\right) \\
& \times \prod_{a=1}^{k+1}\left\{\left(\prod_{i=j_{a-1}+1}^{i_{a}-1} \alpha\left(\lambda_{\bar{\tau}(l)}\right)^{M-m_{i}^{\prime}} \bar{\alpha}\left(\lambda_{\bar{\tau}(i)}\right)^{m_{i}^{\prime}}\right)\right. \\
& \left.\times\left(\prod_{i=i_{a}}^{j_{a}-1} \alpha\left(\lambda_{\bar{\tau}(i)}\right)^{M-m_{i+1}^{\prime}} \bar{\alpha}\left(\lambda_{\tilde{\tau}(i)}\right)^{m_{i+1}^{\prime}}\right)\right\} \\
& \times \prod_{a=1}^{k}\left(\alpha\left(\lambda_{\bar{\tau}\left(J_{a}\right)}\right)^{M-n_{a}} \bar{\alpha}\left(\lambda_{\bar{\tau}\left(J_{a}\right)}\right)^{n_{a}}\right) a_{m_{1}^{\prime}}^{*} \ldots a_{m_{N}^{\prime}}^{*} \Omega .
\end{aligned}
$$

(We have defined $j_{0}=0$ and $j_{k+1}=N$.) Next we can carry out the sums over $n_{1}, \ldots, n_{k}$ : Writing

$$
\zeta_{a}=\frac{\alpha\left(\lambda_{N}\right)}{\bar{\alpha}\left(\lambda_{N}\right)} \frac{\bar{\alpha}\left(\lambda_{\bar{\tau}\left(J_{a}\right)}\right)}{\alpha\left(\lambda_{\bar{\tau}\left(J_{a}\right)}\right)},
$$

we have

$$
\begin{aligned}
\kappa \Delta \sum_{n_{a}=m_{j_{a}}^{\prime}+1}^{m_{j_{a}+1}^{\prime}-1} \zeta_{a}^{n_{a}} & =\kappa \Delta \frac{\zeta_{a}^{m_{j_{a}+1}^{\prime}}-\zeta_{a}^{m_{J_{a}}^{\prime}+1}}{\zeta_{a}-1} \\
& \approx \frac{\kappa}{i\left(\lambda_{\bar{\tau}\left(j_{a}\right)}-\lambda_{N}\right)}\left\{\zeta_{a}^{m_{J_{a}+1}^{\prime}}-\zeta_{a}^{m_{j_{a}}^{\prime}+1}\right\},
\end{aligned}
$$

where we have used the fact that

$$
\zeta_{a} \approx 1+i\left(\lambda_{\bar{\tau}\left(J_{a}\right)}-\lambda_{N}\right) \Delta+O\left(\Delta^{2}\right) .
$$


Inserting (5.50) into (5.48) we obtain

$$
\begin{aligned}
& \Psi_{k}^{(N)} \approx(-i \sqrt{\kappa})^{N} \sum_{1 \leqq m_{1}^{\prime}<\cdots<m_{N}^{\prime} \leqq M} \sum_{1 \leqq l_{1}<\cdots<i_{k+1} \leqq N} \sum_{j_{1}=i_{1}}^{i_{2}-1} \cdots \sum_{j_{k}=i_{k}}^{i_{k+1}-1} \\
& \times \sum_{\tau \in \mathscr{S}_{N-1}} C_{\tau}\left(\lambda_{1}, \ldots, \lambda_{N-1}\right) \alpha\left(\lambda_{N}\right)^{M-\left(m_{i_{1}}^{\prime}+\cdots+m_{i_{k+1}}^{\prime}\right)} \bar{\alpha}\left(\lambda_{N}\right)^{\left(m_{i_{1}}^{\prime}+\cdots+m_{i_{k+1}}^{\prime}\right)} \\
& \times \prod_{a=1}^{k+1}\left\{\left(\prod_{i=j_{a-1}+1}^{i_{a}-1} \alpha\left(\lambda_{\bar{\tau}(i)}\right)^{M-m_{i}^{\prime}} \bar{\alpha}\left(\lambda_{\bar{\tau}(i)}\right)^{m_{i}^{\prime}}\right)\right. \\
& \left.\times\left(\prod_{i=i_{a}}^{j_{a}-1} \alpha\left(\lambda_{\bar{\alpha}(l)}\right)^{M-m_{i+1}^{\prime}} \bar{\alpha}\left(\lambda_{\bar{\tau}(i)}\right)^{m_{i+1}^{\prime}}\right)\right\} \\
& \times \prod_{a=1}^{k}\left\{\left(\alpha\left(\lambda_{N}\right) \bar{\alpha}\left(\lambda_{N}\right)^{-1} \alpha\left(\lambda_{\bar{\tau}\left(j_{a}\right)}\right)^{-1} \bar{\alpha}\left(\lambda_{\bar{\tau}\left(j_{a}\right)}\right)\right)^{m_{j_{a}+1}^{\prime}}\right. \\
& \left.-\left(\alpha\left(\lambda_{N}\right) \bar{\alpha}\left(\lambda_{N}\right)^{-1} \alpha\left(\lambda_{\bar{\tau}\left(j_{a}\right)}\right)^{-1} \bar{\alpha}\left(\lambda_{\bar{\tau}\left(j_{a}\right)}\right)\right)^{m_{j_{a}}^{\prime}+1}\right\} \\
& \times \prod_{a=1}^{k} \frac{\kappa}{i\left(\lambda_{\bar{\tau}\left(j_{a}\right)}-\lambda_{N}\right)} \alpha\left(\lambda_{\bar{\tau}\left(J_{a}\right)}\right)^{M} a_{m_{1}^{\prime}}^{*} \ldots a_{m_{N}}^{*} \Omega .
\end{aligned}
$$

We now split this sum into three parts according to (5.42): We consider separately the terms with given $j_{1}, \ldots, j_{k}$ and for each $a=1, \ldots, k$ we choose either the term $\zeta_{a^{j_{a}+1}}^{m_{1}}$ indicated with a "+ " or the term $\zeta_{a}^{m_{j_{a}+1}}$ indicated with a "-.". A general term of the sum (5.52) can then be labelled with $\left(\left(j_{1}, \pm\right), \ldots,\left(j_{k}, \pm\right)\right)$. The subdivision (5.42) will be as follows:

1. $\Psi_{k, 0}^{(N)}$ contains the terms $\left(\left(i_{1},-\right), \ldots,\left(i_{s},-\right),\left(i_{s+2}-1,+\right), \ldots,\left(i_{k+1}-1,+\right)\right)$ for some $s=0,1, \ldots, k$.

2. $\Psi_{k,}^{(N)}$ - consists of the terms for which there exists $s=1,2, \ldots, k-1$ such that $\left(j_{1}, \pm\right) \in\left\{\left(i_{1},-\right),\left(i_{2}-1,+\right)\right\}, \ldots,\left(j_{s-1}, \pm\right) \in\left\{\left(i_{s-1},-\right),\left(i_{s}-1,+\right)\right\},\left(j_{s}, \pm\right)=$ $\left(i_{s+1}-1,+\right), \quad\left(j_{s+1}, \pm\right)=\left(i_{s+1},-\right) \quad$ and $j_{s+2} \in\left\{i_{s+2}, \ldots, i_{s+3}-1\right\}, \ldots$, $j_{k} \in\left\{i_{k}, \ldots, i_{k+1}-1\right\}$ arbitrary. We shall assume that $s$ is minimal so that if, for some $a=2, \ldots, s-1,\left(j_{a-1}, \pm\right)=\left(i_{a}-1,+\right)$ then $\left(j_{a}, \pm\right) \neq\left(i_{a},-\right)$.

3. $\Psi_{k,+}^{(N)}$ consists of the remaining terms, i.e. those for which there exists $s \in\{1, \ldots, k\}$ such that $\left(j_{s}, \pm\right) \notin\left\{\left(i_{s},-\right),\left(i_{s+1}-1,+\right)\right\}$ and if $\left(j_{a-1}, \pm\right)$ $=\left(i_{a}-1,+\right)$ then $j_{a} \neq\left(i_{a},-\right)$ for $a \in\{1, \ldots, s-1\}$. Again we assume that $s$ is minimal so that $\left.\left(j_{a}, \pm\right) \in\left(i_{a},-\right),\left(i_{a+1}-1,+\right)\right\}$ for $a=1, \ldots, s-1$.

The proof of (5.44)-(5.46) is straightforward. We have

$$
\begin{aligned}
\Psi_{k, 0}^{(N) \approx} & (-i \sqrt{\kappa})^{N} \sum_{1 \leqq m_{1}<m_{N} \leqq M \quad 1 \leqq i_{1}<\cdots<i_{k+1} \leqq N} \\
& \sum_{\tau \in \mathscr{S}_{N-1}} C_{\tau}\left(\lambda_{1}, \ldots, \lambda_{N-1}\right) \sum_{s=0}^{k}(-1)^{s} \alpha\left(\lambda_{N}\right)^{M-m_{t_{s+1}}} \bar{\alpha}\left(\lambda_{N}\right)^{m_{t_{s+1}}} \\
& \times\left(\prod_{i=1}^{i_{s+1}-1} \alpha\left(\lambda_{\bar{\tau}(i)}\right)^{M-m_{i}} \bar{\alpha}\left(\lambda_{\bar{\tau}(i)}\right)^{m_{i}}\right) \\
& \times\left(\prod_{i=i_{s+1}}^{N-1} \alpha\left(\lambda_{\bar{\tau}(i)}\right)^{M-m_{i+1}} \bar{\alpha}\left(\lambda_{\bar{\tau}(i)}\right)^{m_{i+1}}\right) \\
& \times \prod_{a=1}^{s} \frac{\kappa}{i\left(\lambda_{\bar{\tau}\left(i_{a}\right)}-\lambda_{N}\right)} \prod_{a=s+1}^{k} \frac{\kappa}{i\left(\lambda_{\bar{\tau}\left(i_{a+1}-1\right)}-\lambda_{N}\right)} a_{m_{1}}^{*} \ldots a_{m_{N}}^{*} \Omega .
\end{aligned}
$$


If we now define $\sigma \in \mathscr{S}_{N}$ by

$$
\left\{\begin{aligned}
\bar{\sigma}(i) & =\bar{\tau}(i) \quad \text { if } i<i_{s+1}, \\
\bar{\sigma}\left(i_{s+1}\right) & =N \\
\bar{\sigma}(i) & =\bar{\tau}(i-1) \quad \text { if } i>i_{s+1},
\end{aligned}\right.
$$

then it is easy to see that $\tau=\sigma^{\prime}$ as defined by (5.38). Thus we obtain

$$
\begin{aligned}
& \Psi_{k, 0}^{(N)} \approx(-i \sqrt{\kappa})^{N} \sum_{1 \leqq m_{1}<\cdots<m_{N} \leqq M} \sum_{1 \leqq i_{1}<\cdots<i_{k+1} \leqq N} \\
& \sum_{\sigma \in \mathscr{S}_{N}} \prod_{\substack{a=1 \\
i_{a} \neq \sigma(N)}}^{k+1}\left(-\frac{\kappa}{i\left(\lambda_{\bar{\sigma}\left(i_{a}\right)}-\lambda_{N}\right)} \varepsilon_{l_{a}, \sigma(N)}\right) \\
& \times \prod_{i=1}^{N} \alpha\left(\lambda_{\bar{\sigma}(l)}\right)^{M-m_{i}} \bar{\alpha}\left(\lambda_{\bar{\sigma}(i)}\right)^{m_{i}} a_{m_{1}}^{*} \ldots a_{m_{N}}^{*} \Omega \\
& =\sum_{1 \leqq m_{1}<} \sum_{\cdots<m_{N} \leqq M} \sum_{\sigma \in \mathscr{S}_{N} I \subset\{1, \ldots, N\} \backslash \sigma(N)} \prod_{i \in I} \prod_{i \in=k}\left(-\frac{\kappa}{i\left(\lambda_{\bar{\sigma}(l)}-\lambda_{N}\right)} \varepsilon_{i, \sigma(N)}\right) \\
& \times \prod_{i=1}^{N} \alpha\left(\lambda_{i}\right)^{M-m_{\sigma(i)}} \bar{\alpha}\left(\lambda_{i}\right)^{m_{\sigma(i)}} a_{m_{1}}^{*} \ldots a_{m_{N}}^{*} \Omega,
\end{aligned}
$$

which proves (5.44)-(5.46).

Next we consider the more difficult problem of proving (5.43). Analogous to (5.41) we have

$$
\Psi_{0}^{(N)}=\beta_{M, 1}^{*}\left(\lambda_{N}\right) \Psi\left(\lambda_{1}, \ldots, \lambda_{N-1}\right)=\Psi_{0,0}^{(N)}
$$

so that $\Psi_{0,-}^{(N)}=\Psi_{0,+}^{(N)}=0$. Trivially, $\Psi_{1,-}^{(N)}=0$. Finally also $\Psi_{N-1,+}^{(N)}=0$ because, if $k=N-1$ then $i_{1}=1, \ldots, i_{N}=N$ and hence

$\left(j_{a}, \pm\right)=(a, \pm) \in\left\{\left(i_{a},-\right),\left(i_{a+1}-1,+\right)\right\}$ for all $a=1, \ldots, N-1$. To prove that $\Psi_{k,-}^{(N)}+\Psi_{k-1,+}^{(N)}=0$ we write

$$
\Psi_{k,-}^{(N)}=\sum_{s=1}^{k-1} \sum_{r=0}^{s-1} \Psi_{k,-}^{(N)}(r, s),
$$

where $s$ is given in the definition of $\Psi_{k,-}^{(N)}$ and $r$ is such that $\left(j_{a}, \pm\right)=\left(i_{a},-\right)$ for $a=1, \ldots, r$ and $\left(j_{a}, \pm\right)=\left(i_{a+1}-1,+\right)$ for $a=r+1, \ldots, s-1$. Similarly we write

$$
\Psi_{k-1,+}^{(N)}=\sum_{s=1}^{k-1} \sum_{r=0}^{s-1} \Psi_{k-1,+}^{(N)}(r, s),
$$

where $s$ is given in the definition of $\Psi_{k-1,+}^{(N)}$ and $\left(j_{a}, \pm\right)=\left(i_{a},-\right)$ for $a=1, \ldots, r$ and $\left(j_{a}, \pm\right)=\left(i_{a+1}-1,+\right)$ for $a=r+1, \ldots, s-1$. Using the short-hand

$$
\eta_{i}=\left(\alpha\left(\lambda_{i}\right)\right)^{-1} \bar{\alpha}\left(\lambda_{i}\right)
$$


we have

$$
\begin{aligned}
& \Psi_{k,-}^{(N)}(r, s) \approx-i(\sqrt{\kappa})^{N} \sum_{1 \leqq m_{1}<\cdots<m_{N} \leqq M} \sum_{1 \leqq i_{1}<\cdots<i_{k+1} \leqq N} \sum_{j_{s+2}=i_{s+2}}^{i_{s+3}-1} \cdots \sum_{j_{k}=i_{k}}^{i_{k+1}-1} \\
& \sum_{\tau \in \mathscr{S}_{N-1}} C_{\tau}\left(\lambda_{1}, \ldots, \lambda_{N-1}\right)(-1)^{r+1} \prod_{i=1}^{N} \alpha\left(\lambda_{i}\right)^{M} \\
& \left.\times \eta_{N}^{\left(m_{i_{r+1}}\right.}+m_{i_{s+2}}+\cdots+m_{i_{k+1}}\right)-m_{i_{s+1}}\left(\prod_{i=1}^{i_{r+1}-1} \eta_{\bar{\tau}(i)}^{m_{i}}\right)\left(\prod_{i=i_{r+1}}^{i_{s+1}-1} \eta_{\bar{\tau}(l)}^{m_{1+1}}\right) \eta_{\bar{\tau}\left(i_{s+1}\right)}^{m_{i_{s+1}}} \\
& \times \prod_{a=s+2}^{k+1}\left\{\left(\prod_{i=j_{a}-1+1}^{i_{a}-1} \eta_{\bar{\tau}(i)}^{m_{i}}\right)\left(\prod_{i=i_{a}}^{j_{a}-1} \eta_{\bar{\tau}(i)}^{m_{i+1}}\right)\right\} \\
& \times \prod_{a=1}^{r} \frac{\kappa}{i\left(\lambda_{\bar{\tau}\left(l_{a}\right)}-\lambda_{N}\right)} \prod_{a=r+1}^{s} \frac{\kappa}{i\left(\lambda_{\bar{\tau}\left(i_{a+1}-1\right)}-\lambda_{N}\right)} \cdot \frac{\kappa}{i\left(\lambda_{\bar{\tau}\left(i_{s+1}\right)}-\lambda_{N}\right)} \\
& \times \prod_{a=s+2}^{k}\left\{\frac{\kappa}{i\left(\lambda_{\bar{\tau}\left(j_{a}\right)}-\lambda_{N}\right)}\left[\zeta_{a}^{m_{j_{a}+1}}-\zeta_{a}^{m_{j_{a}}+1}\right]\right\} a_{m_{1}}^{*} \ldots a_{m_{N}}^{*} \Omega .
\end{aligned}
$$

(Here $j_{s+1}=i_{s+1}$.) Next we compute $\Psi_{k-1,+}^{(N)}(r, s)$ :

$$
\begin{aligned}
& \Psi_{k-1,+}^{(N)}(r, s) \approx(-i \sqrt{\kappa})^{N} \sum_{1 \leqq m_{1}<\cdot} \sum_{<m_{N} \leqq M} \sum_{1 \leqq i_{1}<\cdot<i_{k} \leqq N}^{i_{s+1}-1} \\
& \times \sum_{j_{s+1}=i_{s+1}}^{i_{s+2}-1} \cdots \sum_{j_{k-1}=i_{k-1}}^{i_{k}-1} \sum_{\tau \in \mathscr{S}_{N-1}} C_{\tau}\left(\lambda_{1}, \ldots, \lambda_{N-1}\right)(-1)^{r} \prod_{i=1}^{N} \alpha\left(\lambda_{i}\right)^{M}
\end{aligned}
$$

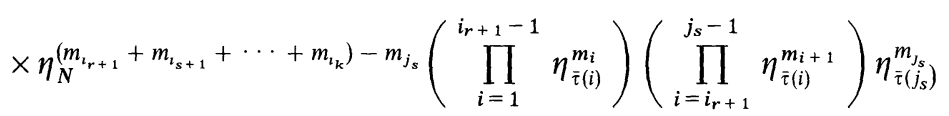

$$
\begin{aligned}
& \times \prod_{a=s+1}^{k}\left\{\left(\prod_{i=j_{a-1}+1}^{i_{a}-1} \eta_{\bar{\tau}(l)}^{m_{2}}\right)\left(\prod_{i=i_{a}}^{j_{a}-1} \eta_{\bar{\tau}(i)}^{m_{i+1}}\right)\right\} \prod_{a=1}^{r} \frac{\kappa}{i\left(\lambda_{\bar{\tau}\left(i_{a}\right)}-\lambda_{N}\right)} \\
& \times \prod_{a=r+1}^{s-1} \frac{\kappa}{i\left(\lambda_{\bar{\tau}\left(i_{a+1}-1\right)}-\lambda_{N}\right)}\left\{\frac{\kappa}{i\left(\lambda_{\bar{\tau}\left(J_{s}-1\right)}-\lambda_{N}\right)}-\frac{\kappa}{i\left(\lambda_{\bar{\tau}\left(j_{s}\right)}-\lambda_{N}\right)}\right\} \\
& \times \prod_{a=s+1}^{k-1}\left\{\frac{\kappa}{i\left(\lambda_{\bar{\tau}\left(j_{a}\right)}-\lambda_{N}\right)}\left[\zeta_{a}^{m_{j_{a}+1}}-\zeta_{a}^{m_{j_{a}}}\right]\right\} a_{m_{1}}^{*} \ldots a_{m_{N}}^{*} \Omega .
\end{aligned}
$$

In this sum we can combine the permutations $\tau$ and $\tilde{\tau}$ which differ only in a transposition of $\bar{\tau}\left(j_{s}\right)$ and $\bar{\tau}\left(j_{s}-1\right)$. The coefficients of $C_{\tau}$ and $C_{\tilde{\tau}}$ differ only in a sign. We can therefore use the fact that

$$
\frac{i\left(\lambda_{\bar{\tau}\left(j_{s}\right)}-\lambda_{\bar{\tau}\left(j_{s}-1\right)}\right)}{\kappa}\left(C_{\tau}-C_{\tilde{\tau}}\right)=C_{\tau}+C_{\tilde{\tau}}
$$


to rewrite $(5.60)$ in the form

$$
\begin{aligned}
& \Psi_{k-1,+}^{(N)} \approx(-i \sqrt{\kappa})^{N} \sum_{1 \leqq m_{1}<\cdots<m_{N} \leqq M} \sum_{1 \leqq i_{1}<\cdots<i_{k} \leqq N} \sum_{j_{s}=i_{s}+1}^{i_{s+1}-1} \\
& \sum_{j_{s+1}=i_{s}+1}^{i_{s+2}-1} \cdots \sum_{j_{k-1}=i_{k-1}}^{i_{k}-1} \sum_{\tau \in \mathscr{S}_{N-1}} C_{\tau}\left(\lambda_{1}, \ldots, \lambda_{N-1}\right)(-1)^{r} \prod_{i=1}^{N} \alpha\left(\lambda_{i}\right)^{M} \\
& \left.\times \eta_{N}^{\left(m_{i+1}\right.}+m_{i_{s+1}}+\cdots+m_{i_{k}}\right)-m_{j_{s}}\left(\prod_{i=1}^{i_{r+1}-1} \eta_{\bar{\tau}(l)}^{m_{i}}\right)\left(\prod_{i=i_{r+1}}^{j_{s}-1} \eta_{\bar{\tau}(i)}^{m_{i+1}}\right) \eta_{\bar{\tau}\left(j_{s}\right)}^{m_{s_{s}}} \\
& \times \prod_{a=s+1}^{k}\left\{\left(\prod_{i=j_{a}-1+1}^{i_{a}-1} \eta_{\bar{\tau}(i)}^{m_{i}}\right)\left(\prod_{i=i_{a}}^{j_{a}-1} \eta_{\bar{\tau}(l)}^{m_{i+1}}\right)\right\} \prod_{a=1}^{r} \frac{\kappa}{i\left(\lambda_{\bar{\tau}\left(l_{a}\right)}-\lambda_{N}\right)} \\
& \times \prod_{a=r+1}^{s-1} \frac{\kappa}{i\left(\lambda_{\bar{\tau}\left(i_{a+1}-1\right)}-\lambda_{N}\right)} \frac{\kappa}{i\left(\lambda_{\bar{\tau}\left(J_{s}-1\right)}-\lambda_{N}\right)} \frac{\kappa}{i\left(\lambda_{\bar{\tau}\left(J_{s}\right)}-\lambda_{N}\right)} \\
& \times \prod_{a=s+1}^{k-1}\left\{\frac{\kappa}{i\left(\lambda_{\bar{\tau}\left(j_{a}\right)}-\lambda_{N}\right)}\left[\zeta_{a^{j_{a}+1}}^{m}-\zeta_{a^{j_{a}}+1}^{m}\right]\right\} a_{m_{1}}^{*} \ldots a_{m_{N}}^{*} \Omega .
\end{aligned}
$$

Comparing this with (5.59) it is now clear that

$$
\Psi_{k,-}^{(N)}(r, s)+\Psi_{k-1,+}^{(N)}(r, s)=0,
$$

which completes the proof of (5.43).

Convergence of the lattice approximation now follows easily:

Theorem 5.1. For arbitrary $N$, the eigenstates $\Psi\left(\lambda_{1}, \ldots, \lambda_{N}\right)$ of the KorepinIzergin lattice model defined by (4.13), where $\left(\lambda_{1}, \ldots, \lambda_{N}\right)$ is a solution of (4.38) respectively (4.39) for a given set of integers $m_{1}<\cdots<m_{N}$ converges to the Bethe Ansatz eigenstate $\Psi_{\left\{k_{j}\right\}}^{B A}$, where $\left(k_{1}, \ldots, k_{N}\right)$ is the solution of (3.2) respectively (3.3) with the same set of integers $m_{1}<\cdots<m_{N}$ :

$$
\lim _{M \rightarrow \infty} \Psi\left(\lambda_{1}, \ldots, \lambda_{N}\right)=(-i \sqrt{\kappa})^{N} e^{-i\left(k_{1}+\cdots+k_{N}\right) / 2} \psi_{\left\{k_{j}\right\}}^{B A} \quad \text { in } \mathscr{F}_{N} \text {-norm } .
$$

Hence the Bethe Ansatz eigenstates for different sets $m_{1}<\cdots<m_{N}$ and $m_{1}^{\prime}<\cdots<m_{N}^{\prime}$ are orthogonal and the set of all Bethe Ansatz eigenstates for an arbitrary set of integers $m_{1}<\cdots<m_{N}$ is complete in $\mathscr{F}_{N}$.

Proof. By Lemma 4.1, $\lambda_{j}(M) \rightarrow k_{j}$ as $M \rightarrow \infty$. Writing $x_{j}=\Delta n_{j}$ in (5.40) we have

$$
\alpha\left(\lambda_{j}\right)^{M-n_{j}} \bar{\alpha}\left(\lambda_{j}\right)^{n_{j}} \rightarrow e^{-i \lambda_{j} / 2} e^{i \lambda_{j}} x^{j} \quad \text { as } M \rightarrow \infty .
$$

By the simple fact that $\sum_{\sigma \in \mathscr{S}_{N}}\left|C_{\sigma}\left(\lambda_{1}, \ldots, \lambda_{N}\right)\right|$ is bounded as $M \rightarrow \infty$ and $\left\|\sum_{1 \leqq n_{1}<\ldots<n_{N} \leqq M} a_{n_{1}}^{*} \ldots a_{n_{N}}^{*} \Omega\right\|^{2} \leqq 1$ we may conclude that (5.64) holds. The orthogonality of the Bethe Ansatz eigenstates then follows from Lemma 4.2 and the completeness from Theorem 3.1.

Acknowledgement. The author wishes to thank Prof. John Lewis and Dr. Joe Pule for stimulating discussions on the subject matter of this paper. The idea of using the Wronskian for the proof of orthogonality (Lemma 4.2) originates from discussions with Dr. Reinhard Werner, which are also gratefully acknowledged. Finally, the author thanks Prof. Brian Davies for inviting him to King's College, London, and explaining to him the proof of norm resolvent convergence mentioned in the remark following Lemma 3.5 . 


\section{References}

1. Babbitt, D., Thomas, L.E.: Commun. Math. Phys. 54, 255-278 (1977)

2. Babbitt, D., Thomas, L.E.: J. Math. Phys. 19, 1699-1704 (1978)

3. Babbit, D., Thomas, L.E.: J. Math. An. \& Appl. 72, 305-328 (1979)

4. Babbit, D., Thomas, L.E., Gutkin.: Lett. Math. Phys. 20, $91-99$ (1990)

5. Baxter, R.J.: Ann. Phys. 70, 323-337 (1972)

6. Baxter, R.J.: Exactly Solved Models in Statistical Mechanics. New York: Academic Press 1982

7. Berg, M. van den, Lewis, J.T., Pulé, J.V.: Commun. Math. Phys. 118, 61-85 (1988)

8. Berg, M. van den, Dorlas, T.C., Lewis, J.T., Pulé, J.V.: Commun. Math. Phys. 127, 41-69 (1990)

9. Berg, M. van den, Dorlas, T.C., Lewis, J.T., Pulé, J.V.: Commun. Math. Phys. 128, 231-245 (1990)

10. Bergknoff, H., Thacker, H.B.: Phys. Rev. D19, 3666-3681 (1979)

11. Bethe, H.: Zeits. f. Phys. 71, 205-226 (1931)

12. Craemer, D.B., Thacker, H.B., Wilkinson, D.: Phys. Rev. D21, 1523-1528 (1980)

13. Destri, C., Lowenstein, J.H.: Nucl. Phys. B205, 369-385 (1982)

14. De Vega, H.J.: Int. J. Mod. Phys. A4, 2371-2463 (1989)

15. Dorlas, T.C., Lewis, J.T., Pulé, J.V.: Commun. Math. Phys. 124, 365-402 (1989)

16. Faddeev, L.D.: Sov. Sc. Rev. C1, 107-155 (1980)

17. Faddeev, L.D., Sklyanin, E.K., Takhtadzhyan, L.A.: Theor. Math. Phys. 40 (1979)

18. Faddeev, L.D., Takhtadzhyan, L.A.: Russ. Math. Surv. 1

19. Gaudin, M.: La fonction d'onde de Bethe, Paris: Masson, 1983

20. Girardeau, M.: J. Math. Phys. 1, 516-523 (1960)

21. Izergin, A.G., Korepin, V.E.: Sov. Phys. Dokl. 26, 653-654 (1981)

22. Izergin, A.G., Korepin, V.E.: Nucl. Phys. B205 [FS5], 401-413 (1982)

23. Izergin, A.G., Korepin, V.E., Smirnov, F.A.: Theor. Math. Phys. 48, 773-776 (English) (1981)

24. Korepin, V.E.: Commun. Math. Phys. 86, 391-418 (1982)

25. Landau, L.D., Lifschitz, E.M.: Statistical Physics, Vol. 5 of: 'Course of Theoretical Physics,' London: Pergamon Press 1958

26. Lieb, E.H., Liniger, W.: Phys. Rev. 130, 1605-1624 (1963)

27. Reed, M., Simon, B.: Methods of Modern Mathematical Physics I: Functional Analysis. (Revised and enlarged edition), New York: Academic Press 1980

28. Reed, M., Simon, B.: Methods of Modern Mathematical Physics II: Fourier Analysis and Self-Adjointness. New York: Academic Press 1975

29. Reed, M., Simon, B.: Methods of Modern Mathematical Physics IV: Analysis of Operators. New York: Academic Press 1978

30. Simon, B.: J. Funct. Anal. 28, 377-385 (1978)

31. Sklyanin, E.K.: Sov. Phys. Dokl. 24, 107-109 (1979)

32. Takahashi, M.: Progr. Theor. Phys. 46, 401-415 (1971)

33. Thacker, H.B., Wilkinson, D.: Phys. Rev. D19, 3660-3665 (1979)

34. Thacker, H.B.: Rev. Mod. Phys. 53, 253-285 (1981)

35. Thomas, L.E.: J. Math. Anal. \& Appl. 59, 392-414 (1977)

36. Yang, C.N., Yang, C.P.: J. Math. Phys. 10, 1115-1122 (1969)

Communicated by B. Simon 